ANNALES

POLONICI MATHEMATICI

$89.1(2006)$

\title{
Opérateurs pseudo-différentiels définis en un point
}

\author{
par Ryuichi Ishimura (Chiba)
}

\begin{abstract}
We introduce the notion of pseudo-differential operators defined at a point and we establish a canonical one-to-one correspondence between such an operator and its symbol. We also prove the invertibility theorem for special type operators.
\end{abstract}

1. Introduction. Dans l'article [9], nous avons introduit la notion des opérateurs pseudo-différentiels non-locaux et leurs symboles et nous avons démontré la correspondance entre un opérateur et son symbole ainsi que le théorème d'inversibilité pour de tels opérateurs. Cette classe d'opérateurs contient les opérateurs aux dérivées partielles eventuellement d'ordre infini, les opérateurs différentiels-différence et les opérateurs de convolution au noyau hyperfonction à support compact. Elle donne une version holomorphe de la théorie des opérateurs pseudo-différentiels d'Hörmander ([6]). En utilisant ces résultats, dans [10], nous avons étudié le prolongement analytique et l'existence d'une solution holomorphe d'une équation pseudodifférentielle non-locale et enfin nous avons établi un calcul opérationnel pour des équations différentielles non-locales à coefficients constants, surtout des équations différentielles-différence à coefficients constants.

Par contre, la classe ainsi établie des opérateurs non-locaux ne peut pas contenir des opérateurs différentiels d'ordre infini aux symboles fonctions entières du type exponentiel changeant avec le point considéré, comme par exemple $e^{x D}$.

Par ailleurs, dans [7], nous avons introduit les opérateurs différentiels, eventuellement d'ordre infini, définis en un point comme les morphismes continus d'un fibre du faisceau des germes de fonctions holomorphes en le point considéré.

Dans le présent article, nous allons définir des opérateurs pseudo-différentiels défini en un point du fibré cotangent $T^{*} X$, ce qui généralise des opéra-

2000 Mathematics Subject Classification: 32W25, 35R50.

Key words and phrases: non-local differential operators, pseudodifferential operators of infinite order, pseudodifferential operators at a point. 
teurs pseudo-différentiels non-locaux et à la fois des opérateurs différentiels définis en un point. Pour un opérateur ainsi généralisé, nous définirons son symbole et nous démontrerons une correspondance canonique entre la classe des opérateurs pseudo-différentiels définis en un point et la classe de leurs symboles. Nous démontrerons qu'un opérateur pseudo-différentiel à un symbole du type spécial admet un inverse dans l'anneau des opérateurs pseudodifférentiels en le point envisagé.

2. Notations. Dans cette section, nous rappelons quelques notations de [9] et [10]. Dans cet article, pour simplicité, on pose $X:=\mathbb{C}^{n}$ et désigne par $\mathcal{O}_{X}$ le faisceau des fonctions holomorphes sur $X$. Pour tout compact convexe $M \subset X$, on utilisera deux fonctions d'appui suivantes : pour tout $\zeta=\left(\zeta_{1}, \ldots, \zeta_{n}\right) \in \mathbb{C}^{n}$,

$$
\begin{aligned}
H_{M}(\zeta) & :=\sup _{z \in M} \operatorname{Re}\langle z, \zeta\rangle, \\
I_{M}(\zeta) & :=\inf _{z \in M} \operatorname{Re}\langle z, \zeta\rangle,
\end{aligned}
$$

où $\langle z, \zeta\rangle:=\sum_{j=1}^{n} z_{j} \zeta_{j}$ avec $z=\left(z_{1}, \ldots, z_{n}\right)$, que l'on désignera parfois par $z \cdot \zeta$. Pour un ensemble $C \subset \mathbb{C}^{n}$, soient $C^{0}$ le polaire $\{z \in X \mid \operatorname{Re}\langle z, \zeta\rangle>0$ pour tout $\zeta \in C\}, C^{\text {a }}$ son antipodale $-C=\{-z \mid z \in C\}$ et $\operatorname{conv} C$ l'enveloppe convexe de $C$. Pour tous $A, B \subset X$, on pose $A+B:=\{a+b \mid$ $a \in A, b \in B\}$ et $A-B:=\{a-b \mid a \in A, b \in B\}$. Pour tous $c, d \in \mathbb{C}$, on note $[a, b]:=\{\lambda a+(1-\lambda) b \mid 0 \leq \lambda \leq 1\},] a, b[:=\{\lambda a+(1-\lambda) b \mid 0<\lambda<1\}$ et $[a, a \infty[:=\{\lambda a \mid \lambda \geq 1\}$ etc.

Dans la suite, on fixe un point $p=\left(x_{0}, \xi_{0}\right) \in T^{*} X$.

DÉfinition 2.1. Soit $\mathfrak{M}=(M(x))_{x \in X}$ une famille de compacts convexes de $X$ indexée par $x \in X$ telle que pour tout voisinage ouvert borné $U$ de $x_{0}$, l'ensemble $M=M_{U}:=\operatorname{conv} \overline{\bigcup_{x \in U} M(x)}$ soit compact. Appelons $\mathfrak{M}$ une famille de supports sur $X$ en $x_{0}$. Pour deux familles de supports $\mathfrak{M}=(M(x))$ et $\mathfrak{M}^{\prime}=\left(M^{\prime}(x)\right)$, on écrira $\mathfrak{M}^{\prime} \prec \mathfrak{M}$ si $M(x) \subset M^{\prime}(x)$ pour tout $x \in X$.

Pour une famille de supports $\mathfrak{M}$, on posera

$$
\Delta_{\mathfrak{M}}:=\{(x, y) \in X \times X \mid z:=y-x \in M(x)\} .
$$

Si $M(x)=M$ pour tout $x \in X$, on écrira aussi $\Delta_{M}$ au lieu de $\Delta_{\mathfrak{M}}$. Et $\Delta_{\{0\}}$ sera écrit simplement $\Delta([9])$.

Pour tous $x \in X$ et $\zeta \in \mathbb{C}^{n}$, considérons les demi-espaces ouverts de $X$

$$
\begin{aligned}
\mathcal{H}_{\zeta}(x) & :=\left\{z \in X \mid \operatorname{Re}\langle z, \zeta\rangle>H_{M(x)}(\zeta)\right\}, \\
\mathcal{I}_{\zeta}(x) & :=\left\{z \in X \mid \operatorname{Re}\langle z, \zeta\rangle>I_{M(x)}(\zeta)\right\} .
\end{aligned}
$$




\section{Définition des opérateurs pseudo-différentiels en un point.} Dans tout le reste de l'article, on supposera que $\mathfrak{M}, \mathfrak{M}^{\prime}$ etc. sont toujours des familles comme dans la définition 2.1. Pour tout cône convexe propre fermé $\Gamma \subset X$ à sommet 0 contenu dans $\left\{\xi_{0}\right\}^{0 \mathrm{a}} \cup\{0\}$ et pour $\varepsilon>0$, on pose $\Gamma_{\varepsilon}=\Gamma_{\varepsilon}\left(\xi_{0}\right):=\left(\Gamma+\varepsilon \xi_{0}\right) \cap\left\{\xi_{0}\right\}^{0 a}$. Soit alors $S \subset X \times X$ un ensemble fermé tel que $C_{\Delta}(S) \subset X \times \Gamma_{\varepsilon}, C_{\Delta}(S)$ étant le cône normal de $S$ le long de $\Delta$, qui est un ensemble conique fermé dans $T_{\Delta}(X \times X)$ (voir [13]). On désigne par $q_{i, j}$ la projection $X \times X \times X \rightarrow X \times X$ sur les $i$-ième et $j$-ième composantes : $q_{i, j}\left(x_{1}, x_{2}, x_{3}\right)=\left(x_{i}, x_{j}\right)$. On rappelle la définition d'un ordinal propre ([12, définition 3.1.1]). Un ensemble fermé $S \subset X \times X$ est un ordinal propre si : (i) $S \supset \Delta$, (ii) $q_{1,3}\left(q_{1,2}^{-1} S \cap q_{2,3}^{-1} S\right) \subset S$, (iii) $q_{1,2}^{-1} S \cap q_{2,3}^{-1} S \stackrel{q_{1,3}}{\rightarrow} S$ est une application propre.

DÉfinition 3.1. $\mathfrak{M}=(M(x))_{x \in X}$ étant comme dans la définition 2.1, un ensemble fermé $Z \subset X \times X$ sera dit un semi-ordinal par rapport à $\mathfrak{M}$ ou à support dans $\mathfrak{M}$ s'il existe un ordinal propre $S$ tel que

(0) $S+\Delta \subset S$,

(i) $S+\Delta_{\mathfrak{M}} \subset Z$,

(ii) $q_{1,3}\left(q_{1,2}^{-1} Z \cap q_{2,3}^{-1} Z\right) \subset Z+\Delta_{\mathfrak{M}}$,

(iii) $q_{1,2}^{-1} Z \cap q_{2,3}^{-1} Z \stackrel{q_{1,3}}{\longrightarrow} Z+\Delta_{\mathfrak{M}}$ est une application propre.

Un ensemble fermé $Z \subset X \times X$ sera appelé un $\mathfrak{M}$-ordinal ou bien un ordinal par rapport à (ou à support dans) $\mathfrak{M}$ s'il est l'intersection d'un nombre fini de semi-ordinaux à support dans $\mathfrak{M}$, c'est-à-dire, il existe un nombre fini de semi-ordinaux $Z_{l}(1 \leq l \leq N)$ à support dans $\mathfrak{M}$ tels que $Z=\bigcap_{l=1}^{N} Z_{l}$. Un ensemble ouvert $W \subset X$ sera dit $Z$-ouvert si $\{y \in X \mid(x, y) \in Z, x \in W\}$ $\subset W$, c'est-à-dire, en désignant par $q_{i}$ la $i$-ième projection de $X \times X$ dans $X$, on a $q_{2}\left(Z \cap q_{1}^{-1} W\right) \subset W$.

DÉfinition 3.2. Soient $Z$ et $Z^{\prime}$ deux ordinaux par rapport à $\mathfrak{M}=$ $(M(x))_{x \in X}$ et $\mathfrak{M}^{\prime}=\left(M^{\prime}(x)\right)_{x \in X}$, respectivement. Le couple $\left(Z, Z^{\prime}\right)$ sera dit composable par rapport à un $\mathfrak{M}^{\prime \prime}$-ordinal $Z^{\prime \prime}$ avec $\mathfrak{M}^{\prime \prime}=\left(M^{\prime \prime}(x)\right)_{x \in X}$ si $M^{\prime \prime}(x)$ est un compact convexe avec $M(x)+M^{\prime}(x) \subset M^{\prime \prime}(x)$ pour tout $x \in X$ et de plus $Z^{\prime \prime} \supset Z^{\prime} \circ Z$ et l'application $q_{13}: q_{1,2}^{-1} Z \cap q_{2,3}^{-1} Z^{\prime} \rightarrow Z^{\prime \prime}$ est propre. On désignera $Z^{\prime} \circ Z:=q_{1,3}\left(q_{1,2}^{-1} Z \cap q_{2,3}^{-1} Z^{\prime}\right)$.

DÉfinition 3.3. Soient $Z, Z^{\prime} \subset X \times X$ deux ordinaux par rapport à $\mathfrak{M}, \mathfrak{M}^{\prime}$, respectivement, dont le couple $\left(Z, Z^{\prime}\right)$ soit composable par rapport à un ordinal $Z^{\prime \prime}$. Soient $D_{1}, D_{2}, D_{1}^{\prime}, D_{2}^{\prime}$ quatre voisinages ouverts de $x_{0}$. On dit que $\left(D_{1}, D_{2} ; D_{1}^{\prime}, D_{2}^{\prime}\right)$ est $\left(Z, Z^{\prime}\right)$-rond si $\left\{y \in X \mid(x, y) \in Z,(y, z) \in Z^{\prime}\right.$, $\left.x \in D_{1}, z \in D_{2}^{\prime}\right\} \subset D_{2} \cap D_{1}^{\prime}$, c'est-à-dire, en désignant par $q_{i}$ la $i$-ième projection de $X \times X \times X$ dans $X$, 


$$
q_{2}\left(q_{1,2}^{-1} Z \cap q_{2,3}^{-1} Z^{\prime} \cap q_{1}^{-1} D_{1} \cap q_{3}^{-1} D_{2}^{\prime}\right) \subset D_{2} \cap D_{1}^{\prime} .
$$

Nous dirons simplement $Z$-rond si $\mathfrak{M}=\mathfrak{M}^{\prime}, Z=Z^{\prime}$.

DÉFInition 3.4. Un $\mathfrak{M}$-ordinal $Z$ sera dit dans la direction $\xi_{0}$ s'il y a un ordinal propre correspondant $S$ satisfaisant à

$$
C_{\Delta}(S) \subset X \times \Gamma_{\varepsilon}
$$

avec $\Gamma$ un cône convexe fermé. Pour un M-ordinal $Z \subset X \times X$ dans la direction $\xi_{0}$ et deux voisinages ouverts quelconques $D_{1}, D_{2}$ de $x_{0}$, on définit maintenant

$$
\mathcal{E}\left(Z ; D_{1}, D_{2}\right):=H_{Z}^{n}\left(D_{1} \times D_{2}, \mathcal{O}_{X \times X}^{(0, n)}\right) .
$$

Ici $\mathcal{O}_{X \times X}^{(0, n)}$ signifie le faisceau des $(0, n)$-formes holomorphes.

Remarquons que pour deux familles de supports $\mathfrak{M}$ et $\mathfrak{M}^{\prime}$ avec $\mathfrak{M} \succ \mathfrak{M}^{\prime}$ et ordinaux $Z$ et $Z^{\prime}$ par rapport à $\mathfrak{M}$ et $\mathfrak{M}^{\prime}$, respectivement, et pour quatre ouverts $D_{1}, D_{2}, D_{1}^{\prime}, D_{2}^{\prime}$, si $D_{1} \subset D_{1}^{\prime}, D_{2} \subset D_{2}^{\prime}$ et $Z \supset Z^{\prime}$, alors on a le morphisme canonique

$$
\mathcal{E}\left(Z^{\prime} ; D_{1}^{\prime}, D_{2}^{\prime}\right) \rightarrow \mathcal{E}\left(Z ; D_{1}, D_{2}\right) .
$$

Dans le cas où $M(x)=M$ est un compact convexe fixé pour tout $x \in X$, la définition est une généralisation de celle donnée dans la définition 3.4 de [9] et elle nous permet de prendre $D_{1}, D_{2}$ aussi petits que l'on veut. En effet, dans [9], on était obligé de supposer l'ouvert $D_{2}$ de contenir $D_{1}+M$.

DÉFINITION 3.5. On pose

$$
\mathcal{E}_{[p]}^{\mathbb{R}}:=\underset{\mathfrak{M}, Z, D_{1}, D_{2}}{\lim _{\vec{E}}} \mathcal{E}\left(Z ; D_{1}, D_{2}\right)
$$

où $\mathfrak{M}$ parcourt les familles de supports en $x_{0}$ ordonnées par $\prec, Z$ parcourt les ordinaux dans la direction $\xi_{0}$ par rapport à $\mathfrak{M}$, et $D_{1}, D_{2}$ parcourent les voisinages ouverts de $x_{0}$; on appelera tout $P \in \mathcal{E}_{[p]}^{\mathbb{R}}$ un opérateur pseudodifférentiel en le point $p$.

Proposition 3.6. Soient $Z, Z^{\prime}$ deux ordinaux par rapport à $\mathfrak{M}, \mathfrak{M}^{\prime}$, respectivement, en $\xi_{0}$ tels que $\left(Z, Z^{\prime}\right)$ soit composable par rapport à un $\mathfrak{M}^{\prime \prime}$ ordinal $Z^{\prime \prime}$. Soient $D_{1}, D_{2}, D_{1}^{\prime}, D_{2}^{\prime}$ quatre voisinages ouverts de $x_{0}$ tels que $\left(D_{1}, D_{2} ; D_{1}^{\prime}, D_{2}^{\prime}\right)$ soit $\left(Z, Z^{\prime}\right)$-rond. Alors on a le morphisme naturel suivant que nous appelons la composition:

$$
\mathcal{E}\left(Z ; D_{1}, D_{2}\right) \otimes \mathcal{E}\left(Z^{\prime} ; D_{1}^{\prime}, D_{2}^{\prime}\right) \rightarrow \mathcal{E}\left(Z^{\prime \prime} ; D_{1}, D_{2}^{\prime}\right) .
$$

En particulier, cela munit $\mathcal{E}_{[p]}^{\mathbb{R}}$ de la structure d'anneau. 
Démonstration. On a d'abord, par le "cup-product", le morphisme

$$
\begin{aligned}
\mathcal{E}\left(Z ; D_{1}, D_{2}\right) \otimes \mathcal{E} & \left(Z^{\prime} ; D_{1}^{\prime}, D_{2}^{\prime}\right) \\
& =H_{Z}^{n}\left(D_{1} \times D_{2}, \mathcal{O}_{X \times X}^{(0, n)}\right) \otimes H_{Z^{\prime}}^{n}\left(D_{1}^{\prime} \times D_{2}^{\prime}, \mathcal{O}_{X \times X}^{(0, n)}\right) \\
& \rightarrow H_{q_{1,2}^{-1} Z \cap q_{2,3}^{-1} Z^{\prime}}^{2 n}\left(D_{1} \times\left(D_{2} \cap D_{1}^{\prime}\right) \times D_{2}^{\prime}, \mathcal{O}_{X \times X \times X}^{(0, n, n)}\right) .
\end{aligned}
$$

Comme dans [9], compte tenu de la définition 3.2, on applique la proposition 3.1.4 de [12] :

$$
\begin{aligned}
H_{q_{1,2}^{-1} Z \cap q_{2,3}^{-1} Z^{\prime}}^{2 n}\left(D_{1} \times\left(D_{2} \cap D_{1}^{\prime}\right) \times D_{2}^{\prime}\right. & \left., \mathcal{O}_{X \times X \times X}^{(0, n, n)}\right) \\
& \rightarrow H_{Z^{\prime \prime}}^{2 n}\left(D_{1} \times D_{2}^{\prime}, R q_{1,3 !} \mathcal{O}_{X \times X \times X}^{(0, n, n)}\right) .
\end{aligned}
$$

Enfin, l'intégration le long de fibre $\int_{q_{1,3}} d x_{2}$ donne

$$
H_{Z^{\prime \prime}}^{2 n}\left(D_{1} \times D_{2}^{\prime}, R q_{1,3 !} \mathcal{O}_{X \times X \times X}^{(0, n, n)}\right) \rightarrow H_{Z^{\prime \prime}}^{n}\left(D_{1} \times D_{2}^{\prime}, \mathcal{O}_{X \times X}^{(0, n)}\right) .
$$

Pour tous $P \in \mathcal{E}\left(Z ; D_{1}, D_{2}\right)$ et $Q \in \mathcal{E}\left(Z^{\prime} ; D_{1}^{\prime}, D_{2}^{\prime}\right)$, on désigne par $Q \circ P \in$ $\mathcal{E}\left(Z^{\prime \prime} ; D_{1}, D_{2}^{\prime}\right)$ l'image par ce morphisme et appelons-la composition de $P$ et $Q$.

Proposition 3.7. Pour un $\mathfrak{M}$-ordinal $Z$ en $\xi_{0}$, soient $D_{1}, D_{2}$ deux voisinages ouverts de $x_{0}$ dans $X$. Soient $W, W_{0} \subset X$ deux $Z$-ouverts tels que $W_{0} \subset W$ et $W \backslash W_{0} \subset D_{1}$. Alors, pour tout $k$, on a le morphisme naturel

$$
\mathcal{E}\left(Z ; D_{1}, D_{2}\right) \otimes H_{W \backslash W_{0}}^{k}\left(D_{2} \cap W, \mathcal{O}_{X}\right) \rightarrow H_{W \backslash W_{0}}^{k}\left(D_{1} \cap W, \mathcal{O}_{X}\right) .
$$

Démonstration. Soit $q_{i}$ la $i$-ième projection de $X \times X$ dans $X, i=1,2$. Posons $\Lambda:=q_{2}^{-1}(T) \cap Z$. Alors le "cup-product" donne

$$
\begin{aligned}
\mathcal{E}\left(Z ; D_{1}, D_{2}\right) \otimes H_{T}^{k}\left(D_{2} \cap\right. & \left.W, \mathcal{O}_{X}\right) \\
& =H_{Z}^{n}\left(D_{1} \times D_{2}, \mathcal{O}_{X \times X}^{(0, n)}\right) \otimes H_{T}^{k}\left(D_{2} \cap W, \mathcal{O}_{X}\right) \\
& \rightarrow H_{\Lambda}^{n+k}\left(D_{1} \times\left(D_{2} \cap W\right), \mathcal{O}_{X \times X}^{(0, n)}\right) .
\end{aligned}
$$

Comme dans la preuve précédente, on peut prouver facilement que $q_{1}^{-1}(T) \cap$ $\Lambda$ est un ouvert dans $\Lambda$ et la projection $q_{1 \mid q_{1}^{-1}(T) \cap \Lambda}: q_{1}^{-1}(T) \cap \Lambda \rightarrow T$ est propre. D'où on peut appliquer la proposition 3.1.4 de [12] :

$$
\begin{aligned}
H_{\Lambda}^{n+k}\left(D_{1} \times\right. & \left.\left(D_{2} \cap W\right), \mathcal{O}_{X \times X}^{(0, n)}\right) \\
& \rightarrow H_{T}^{n+k}\left(D_{1}, R q_{1 !} \mathcal{O}_{X \times X}^{(0, n)}\right)=H_{T}^{n+k}\left(D_{1} \cap W, R q_{1 !} \mathcal{O}_{X \times X}^{(0, n)}\right) .
\end{aligned}
$$

Par l'intégration le long de fibre de $q_{1}$, on a

$$
H_{T}^{n+k}\left(D_{1} \cap W, R q_{1 !} \mathcal{O}_{X \times X}^{(0, n)}\right) \rightarrow H_{T}^{k}\left(D_{1} \cap W, \mathcal{O}_{X}\right) .
$$


En particular, dans le cas de $k=1$, on a :

Corollaire 3.8. Dans la situation de la proposition, soit $P \in \mathcal{E}(Z$; $\left.D_{1}, D_{2}\right)$. Alors on a l'opération

$$
P: \mathcal{O}\left(D_{2} \cap W_{0}\right) / \mathcal{O}\left(D_{2} \cap W\right) \rightarrow \mathcal{O}\left(D_{1} \cap W_{0}\right) / \mathcal{O}\left(D_{1} \cap W\right) .
$$

De même, lorsque $p$ est réel, l'opérateur pseudo-différentiel en $p$ opère sur les germes des microfonctions définies en $p$ :

Proposition 3.9. Soit $R:=\mathbb{R}^{n} \subset X=\mathbb{C}^{n}$ et soit $p=\left(x_{0}, \xi_{0}\right)$ réel, c'est-à-dire $p \in T^{*} R$. Désignons par $\mathcal{C}_{R}$ le faisceau des microfonctions sur $R$. Alors on a le morphisme naturel

$$
\mathcal{E}_{[p]}^{\mathbb{R}} \otimes \mathcal{C}_{R, p} \rightarrow \mathcal{C}_{R, p}
$$

Dans la suite, on appliquera la discussion précédente au cas suivant. Soient $\Gamma_{1}, \ldots, \Gamma_{N}$ des cônes propres convexes fermés dans $X$ à sommet 0 . Posons

$$
G(x):=\bigcap_{l=1}^{N}\left(\Gamma_{l}+M(x)\right)
$$

pour tout $x \in X$ et $\mathcal{G}:=(G(x))_{x \in X}$. De plus, on pose $Z=Z_{\mathcal{G}}:=\{(x, y) \in$ $X \times X \mid y-x \in G(x)\}$. Chaque $Z_{l}:=\left\{(x, y) \in X \times X \mid y-x \in \Gamma_{l}+M(x)\right\}$ $(1 \leq l \leq N)$ est un semi-ordinal à support dans $\mathfrak{M}$ et donc $Z=\bigcap_{l=1}^{N} Z_{l}$ est un $\mathfrak{M}$-ordinal au sens de la définition 3.1. Dans ce cas, la notion de $Z$-ouvert ou bien $Z$-rond est simplement redit comme $\mathcal{G}$-ouvert ou $\mathcal{G}$-rond et pour deux voisinages ouverts $D_{1}, D_{2}$ de $x_{0}$, on note

$$
\mathcal{E}\left(\mathcal{G} ; D_{1}, D_{2}\right):=\mathcal{E}\left(Z ; D_{1}, D_{2}\right) .
$$

On peut vérifier facilement que c'est un cas particulier de la définition 3.4 et aussi que toutes les hypothèses des propositions 3.5 et 3.6 sont vérifiés dans ce cas.

En particulier, on a la proposition suivante:

Proposition 3.10. Dans la situation ci-dessus, soient $\mathfrak{M}$ une famille de supports et $Z_{\mathcal{G}}$ le $\mathfrak{M}$-ordinal en $\xi_{0}$ défini par $\mathcal{G}=(G(x))$ donné par (3.2). Soient $D_{1}, D_{2}$ deux voisinages ouverts de $x_{0}$ et soit $P \in \mathcal{E}\left(\mathcal{G} ; D_{1}, D_{2}\right)$. Alors on a l'opération

$$
P: \underset{\lim }{\longrightarrow} \mathcal{O}\left(D \cap W_{0}\right) / \mathcal{O}(D \cap W) \rightarrow \underline{\lim } \mathcal{O}\left(D \cap W_{0}\right) / \mathcal{O}(D \cap W)
$$

où la limite inductive est prise, en posant $\Gamma:=\bigcap_{l=1}^{N} \Gamma_{l}$, pour $D$ parcourant des voisinages ouverts $\Gamma$-ronds de $x_{0}$ et pour $W, W_{0} \subset X$ parcourant des $\Gamma$-ouverts tels que $W_{0} \subset W$ et $W \backslash W_{0} \subset D$. Par suite, la limite inductive a la structure d'un $\mathcal{E}_{[p]}^{\mathbb{R}}$-module.

Dans le reste de cette section, on suppose que $D_{1}, D_{2}$ soient convexes. Prenant des coordonnées sur $X$, on peut reformuler (3.3) dans le langage de 
cohomologie de Čech. Par une rotation de coordonnées, on peut supposer que $\xi_{0}=(1,0, \ldots, 0)$. Pour tout $\delta>0$, prenons les cônes

$$
\begin{aligned}
& \Gamma_{1, \delta}=\Gamma_{1}:=\left\{z \in X|\delta| \operatorname{Im} z_{1} \mid \leq-\operatorname{Re} z_{1}\right\} \\
& \Gamma_{j, \delta}=\Gamma_{j}:=\left\{z \in X|\delta| z_{j}|\leq| z_{1} \mid\right\} \quad(2 \leq j \leq n),
\end{aligned}
$$

et calculons $\mathcal{E}\left(\mathcal{G} ; D_{1}, D_{2}\right)$. Définissons les domaines d'holomorphie suivants :

$$
V_{k}:=D_{1} \times D_{2} \backslash\left\{(x, y) \mid z=y-x \in \Gamma_{k}+M(x)\right\} \quad(1 \leq k \leq n) .
$$

(Il est facile de voir qu'ils sont des domaines d'holomorphie si on applique, par exemple, le théorème 2.6.9 de [5].) Alors pour $Z:=\{(x, y) \in X \times X \mid$ $\left.y-x \in \bigcap_{k=1}^{n}\left(\Gamma_{k}+M(x)\right)\right\}, \mathcal{V}:=\left\{V_{1}, \ldots, V_{n}\right\}$ est un recouvrement ouvert de $D_{1} \times D_{2} \backslash Z$. Désignons $V:=\bigcap_{k=1}^{n} V_{k}$ et $V_{\widehat{k}}:=\bigcap_{l \neq k} V_{l}$ pour $k=1, \ldots, n$. Alors on a $V=D_{1} \times D_{2} \backslash \bigcup_{k=1}^{n}\left\{(x, y) \mid z=y-x \in \Gamma_{k}+M(x)\right\}$. On voit facilement que

$$
H_{Z}^{n}\left(D_{1} \times D_{2}, \mathcal{O}_{X \times X}^{(0, n)}\right) \simeq \frac{\mathcal{O}_{X \times X}^{(0, n)}(V)}{\sum_{k=1}^{n} \mathcal{O}_{X \times X}^{(0, n)}\left(V_{\widehat{k}}\right)} .
$$

D'où, à chaque $P \in \mathcal{E}_{M}(\mathcal{G} ; D)$ correspond une $(0, n)$-forme $K(x, z) d y=$ $K(x, y) d y \in \mathcal{O}_{X \times X}^{(0, n)}(V)$, determinée à $\sum_{k=1}^{n} \mathcal{O}_{X \times X}^{(0, n)}\left(V_{\widehat{k}}\right)$ près, et que nous appelons le noyau de $P$.

Remarque. Dans la situation du corollaire 3.8, l'action de $P$ sur $f(x) \in$ $\mathcal{O}\left(D_{2} \cap W_{0}\right)$, qui est determiné à $\mathcal{O}\left(D_{2} \cap W\right)$ près, est donnée par

$$
P f(x)=\int_{\gamma} K(x, x+z) f(x+z) d z
$$

où le chemin d'intégration $\gamma$ sera défini ultérieurement (voir (4.6)).

4. Symbole d'un opérateur pseudo-différentiel en un point. Nous allons interpréter un opérateur pseudo-différentiel par son symbole. Le raisonnement que l'on emploiera ici a été initié par une note personnelle de K. Kataoka, qui a établi la correspondance ci-dessous en cas des opérateurs pseudo-différentiels (locaux). On peut le retrouver dans le polycopié du cours d'Aoki et Yamazaki [3] et dans le livre d'Aoki, Kataoka et Ymazaki [4].

Dans cette section toute entière, on suppose que $x_{0}=0$ et on fixe une famille de supports $\mathfrak{M}=(M(x))_{x \in X}$ dont on suppose qu'elle satisfait à l'hypothèse suivante :

$$
\max _{m \in M(x)}|m| \rightarrow 0 \quad(|x| \rightarrow 0)
$$

Soit $\Omega$ un voisinage conique de $p=\left(x_{0}, \xi_{0}\right) \in T^{*} X$. Pour tout $r>0$, on désignera

$$
\Omega(r):=\{(x, \xi) \in \Omega|| \xi \mid>r\}, \quad \Omega[r]:=\{(x, \xi) \in \Omega|| \xi \mid \geq r\} .
$$


DÉfinition 4.1 (cf. [9] et [1]). Pour $\mathfrak{M}=(M(x))_{x \in X}, M(x)$ étant compact convexe, on pose

$$
\begin{aligned}
S^{\mathfrak{M}}(\Omega):=\{ & P(x, \xi) \in \mathcal{O}(\Omega(r))(\text { avec } r>0) \mid \\
& \text { pour tout ensemble conique } \Omega^{\prime} \Subset \Omega, \text { tout } r^{\prime}>r \\
& \text { et tout } \varepsilon>0, \text { il existe } C_{\varepsilon}>0 \text { tel que } \\
& \left.|P(x, \xi)| \leq C_{\varepsilon} e^{H_{M(x)}(\xi)+\varepsilon|\xi|} \text { pour tout }(x, \xi) \in \Omega^{\prime}\left[r^{\prime}\right]\right\}
\end{aligned}
$$

et

$$
\begin{aligned}
N^{\mathfrak{M}}(\Omega):= & \left\{P(x, \xi) \in S^{\mathfrak{M}}(\Omega) \mid P(x, \xi) \in \mathcal{O}(\Omega(r))\right. \text {, et } \\
& \text { pour tout ensemble conique } \Omega^{\prime} \Subset \Omega \text { et tout } r^{\prime}>r, \\
& \text { il existe } \varepsilon_{0}>0 \text { et } C>0 \text { tels que } \\
& \left.|P(x, \xi)| \leq C e^{I_{M(x)}(\xi)-\varepsilon_{0}|\xi|} \text { pour tout }(x, \xi) \in \Omega^{\prime}\left[r^{\prime}\right]\right\} .
\end{aligned}
$$

Les symboles que nous considérons ici sont donc des fonctions holomorphes du type exponentiel définies dans l'ensemble conique $\Omega(r)$, son indicatrice de croissance en $\xi$ (voir par exemple [14] ou [15])

$$
h_{P, x}^{*}(\xi):=\limsup _{\Omega(r) \ni \xi^{\prime} \rightarrow \xi} h_{P, x}\left(\xi^{\prime}\right),
$$

avec

$$
h_{P, x}(\xi):=\limsup _{t \rightarrow \infty} \frac{\ln |P(x, t \xi)|}{t},
$$

changeant avec $x$. On remarque que

$$
h_{P, x}^{*}(\xi) \leq H_{M(x)}(\xi) .
$$

On travaillera dans la situation de la dernière partie de la section précédente. En particulier, par un changement de variables, on suppose que $x_{0}=0$, $\xi_{0}=(1,0, \ldots, 0)$. On pose $G(x):=\bigcap_{k=1}^{n}\left(\Gamma_{k}+M(x)\right)$ pour tout $x \in X$ et $\mathcal{G}:=(G(x))_{x \in X}$. Dans la suite, bien que les preuves que nous donnerons soient tout à fait analogues à celles de [9], nous les répétons quand même.

Avec les notations de la section précédente, soit $P \in \mathcal{E}\left(\mathcal{G} ; D_{1}, D_{2}\right)$ où $D_{1}, D_{2}$ sont des voisinages ouverts convexes de $x_{0}=0$, et soit $K(x, y) d y$ un de ses noyaux définies par (3.7). On peut supposer que $D_{1}, D_{2}$ satisfont à la condition suivante : Soit $\pi_{k}$ la $k$-ième projection de $\mathbb{C}^{n}$ à $\mathbb{C}$. Compte tenu de (2.2), (3.6), pour un petit voisinage ouvert $U \subset D_{1}$ de $x_{0}=0$, posons pour tout $x \in U, A_{k}(x):=\pi_{k}\left(\left(\mathcal{I}_{\xi_{0}}(x)+x\right) \cap G(x)\right), A_{k}:=\bigcup_{x \in U} A_{k}(x), I_{1}(x):=$ $\pi_{1}\left(\mathcal{I}_{\xi_{0}}(x)+x\right), I_{1}:=\bigcup_{x \in U} I_{1}(x), B_{1}(x):=\partial A_{1}(x) \backslash \partial I_{1}(x), B_{1}:=\partial A_{1} \backslash \partial I_{1}$, $B_{j}:=\partial\left(\operatorname{conv} A_{j}\right)(2 \leq j \leq n)$ et $B:=B_{1} \times \cdots \times B_{n}$. Alors il existe un ouvert $W \ni 0$ tel que

$$
B+W \subset D_{2}
$$

où $M=M_{U}:=\operatorname{conv} \overline{\bigcup_{x \in U} M(x)}$ est un compact convexe grâce à la définition 2.1. Définissons maintenant un chemin d'intégration $\gamma$ dans $X$ tel que 
pour un ouvert $W_{1} \Subset W$ non-vide, l'ensemble $\left\{(x, x+z) \mid x \in W_{1}, z \in \gamma\right\}$ est inclus dans le domaine $V=\bigcap_{k=1}^{n} V_{k}$ où $K(x, y) d y$ est défini. Il y a d'abord deux points $a, b \in \mathbb{C} \backslash A_{1}$ près de $\partial A_{1}$ tels que $\operatorname{Re} a, \operatorname{Re} b<I_{M}\left(\xi_{0}\right)$ et $\operatorname{Im} a<\inf _{z \in M} \operatorname{Im} z_{1}, \operatorname{Im} b>\sup _{z \in M} \operatorname{Im} z_{1}$. Prenons un chemin lisse de Jordan $\gamma_{1} \subset \mathbb{C}$ orienté positivement ayant $a$ comme point de départ et $b$ comme point final tel que

$$
\widetilde{\gamma}_{1}:=\left\{\left(x, x+\left(z_{1}, \ldots, z_{n}\right)\right) \in W_{1} \times D_{2} \mid z_{1} \in \gamma_{1}, z_{j} \in \mathbb{C}(2 \leq j \leq n)\right\} \subset V_{1} .
$$

Pour $z_{1} \in \gamma_{1}$, soit $\gamma_{j}=\gamma_{j}\left(z_{1}\right) \subset \mathbb{C}$ une courbe lisse fermée de Jordan d'orientation positive telle que

$$
\widetilde{\gamma}_{j}:=\left\{\left(x, x+\left(z_{1}, \ldots, z_{n}\right)\right) \in W_{1} \times D_{2} \mid z_{j} \in \gamma_{j}, z_{i} \in \mathbb{C}(i \neq 1, j)\right\} \subset V_{j} .
$$

(Ceci est possible si l'on prend $U_{1}$ assez petit). Notons que par (4.5), $\gamma_{j}$ pourra être pris indépendamment de $z_{1}$. En posant $\gamma:=\gamma_{1} \times \cdots \times \gamma_{n}$, on définit le symbole de $P$ comme suit :

$$
\sigma(P)(x, \xi):=\int_{\gamma} e^{z \cdot \xi} K(x, x+z) d z .
$$

Proposition 4.2. Dans la situation précédente, on peut supposer (4.5). Alors il existe un voisinage conique $\Omega$ de $p:=\left(x_{0}, \xi_{0}\right)$ tel que $W \subset \pi(\Omega)$, $\pi: T^{*} X \rightarrow X$ étant la projection, et que pour tout $P \in \mathcal{E}\left(\mathcal{G} ; D_{1}, D_{2}\right)$ et tout noyau $K(x, y) d y \in \mathcal{O}_{X \times X}^{(0, n)}(V)$ de $P$, le symbole $\sigma(P)$ de $P$ défini par (4.6) appartienne à $S^{\mathfrak{M}}(\Omega)$. En outre, le symbole $\sigma(P)$ est déterminé dans $S^{\mathfrak{M}}(\Omega)$ modulo $N^{\mathfrak{M}}(\Omega)$.

Démonstration. Exactement comme la démonstration de la proposition 4.2 de [9] : Évidemment $\sigma(P)$ défini par (4.6) satisfait à (4.2) en $p=$ $\left(x_{0}, \xi_{0}\right)$ et par une rotation de variables, on a $\sigma(P) \in S^{\mathfrak{M}}(\Omega)$. Si $\gamma$ est pris près de chaque $M(x)$, on a $\sigma(P) \in N^{\mathfrak{M}}(\Omega)$ lorsque $K(x, y) d y \in \mathcal{O}_{X \times X}^{(0, n)}\left(V_{\widehat{1}}\right)$, et $\sigma(P)=0$ lorsque $K(x, y) d y \in \mathcal{O}_{X \times X}^{(0, n)}\left(V_{\widehat{j}}\right)(2 \leq j \leq n)$.

Ensuite, on prend un symbole $P(x, \xi) \in S^{\mathfrak{M}}(\Omega)$; on y associera le noyau $K(x, y) d y$ d'un opérateur pseudo-différentiel $P$ en $p$ et on démontrera que cette correspondance et celle donnée dans la proposition 4.2 sont réciproques l'une à l'autre.

Soient $W_{1} \subset W$ un ouvert et $\omega$ un cône ouvert à sommet 0 tels que $\omega \ni \xi_{0}$ et $W_{1} \times \omega \subset \Omega$. Pour tout $z \in \mathcal{H}_{\xi}(x)$, il existe $\varepsilon_{0}>0$ tel que $\operatorname{Re}\langle z, \xi\rangle>H_{M(x)}(\xi)+\varepsilon_{0}|\xi|((2.1))$. Alors pour tous $x \in U$ et $\varrho \in \mathbb{C}$ tels que $|\varrho|>r(r$ étant celui de $(4.2))$ et $\varrho \xi \in \omega$, posons

$$
L_{\varrho}(x, z, \xi):=\int_{\varrho}^{\infty} e^{-\tau z \cdot \xi} \tau^{n-1} P(x, \tau \xi) d \tau
$$


où le chemin d'intégration est pris dans la direction [ $\varrho, \varrho \infty\left[\right.$. Par (4.2), si $W_{1}$ est assez petit, cette intégrale sera absolument et compactement convergente $\operatorname{sur}\left\{(x, z, \xi) \in W_{1} \times X \times \omega \mid \operatorname{Re}\langle z, \xi\rangle>H_{M(x)}(\xi)\right\}$. Pour $\eta^{\prime}=\left(\eta_{2}, \ldots, \eta_{n}\right)$ avec $\left|\eta^{\prime}\right| \ll 1$, posons $\eta:=\left(1, \eta^{\prime}\right):=\left(1, \eta_{2}, \eta_{3}, \ldots, \eta_{n}\right)$. Pour tout $\varepsilon>0$ et $z$ avec $z_{j} \neq 0(2 \leq j \leq n)$, on définit la $(n-1)$-chaîne

$$
\widetilde{\beta}:=\left[0, \varepsilon / z_{2}\right] \times\left[0, \varepsilon / z_{3}\right] \times \cdots \times\left[0, \varepsilon / z_{n}\right] .
$$

On définit le noyau de $P(x, \xi)$ par la formule

$$
K_{\varrho}(x, y):=\frac{1}{(2 \pi \sqrt{-1})^{n}}\left[\int_{\widetilde{\beta}} L_{\varrho}(x, z, \eta) d \eta^{\prime}\right]_{\mid z=y-x}
$$

avec $\eta=\left(1, \eta^{\prime}\right)$. Par translation, on peut supposer $0 \in M(x)$ et donc pour tout $z \notin \bigcup_{k=1}^{n}\left(\Gamma_{k}+M(x)\right)$, on a $z_{2}, \ldots, z_{n} \neq 0$. En remarquant qu'une petite oscillation de la direction d'intégrale $[\varrho, \varrho \infty[$ dans (4.7) donne une prolongation analytique, on a aisément que pour $(x, y) \in V$ avec $\delta>0$ dans la notations précédentes (section 3$), K_{\varrho}(x, y)$, qui sera maintenant désigné par $K(x, y)$, est holomorphe. À partir de $P(x, \xi)$, le noyau $K(x, y)$ est en effet déterminé à $\sum_{k=1}^{n} \mathcal{O}_{X \times X}\left(V_{\widehat{k}}\right)$ près, car pour $\varrho^{\prime}$ près de $\varrho$, on a

$$
\begin{aligned}
K_{\varrho}(x, y) & -K_{\varrho^{\prime}}(x, y) \\
= & \frac{1}{(2 \pi \sqrt{-1})^{n}}\left[\int_{\widetilde{\beta}} d \eta^{\prime} \int_{\varrho}^{\varrho^{\prime}} e^{-\tau z \cdot \eta} \tau^{n-1} P(x, \tau \eta) d \tau\right]_{\mid z=y-x} \in \sum_{k=1}^{n} \mathcal{O}_{X \times X}\left(V_{\widehat{k}}\right) .
\end{aligned}
$$

Si $P(x, \xi) \in N^{\mathfrak{M}}(\Omega)$, compte tenu de (4.1), on voit aussi qu'il y a $\delta>0$ tel que, en prenant $D_{1}$ plus petit pour que $D_{2}$ satisfasse à $(4.5)$, on a $K(x, y) \in$ $\sum_{k=1}^{n} \mathcal{O}_{X \times X}\left(V_{\widehat{k}}\right)$. D'où on a

Proposition 4.3. Pour tout voisinage ouvert conique $\Omega \subset T^{*} X$ de $p=\left(0, \xi_{0}\right), \xi_{0}$ étant $(1,0, \ldots, 0)$, il existe des cônes $\Gamma_{1, \delta}, \ldots, \Gamma_{n, \delta}$ de la forme (3.4) et (3.5) avec $\delta>0$ et deux voisinages ouverts convexes $D_{1}, D_{2}$ de $x_{0}$ satisfaisant à (4.5) avec un ouvert $W \subset D_{1}$ qui n'est pas vide tel qu'à tout symbole $P(x, \xi) \in S^{\mathfrak{M}}(\Omega)$, on associe le noyau $K(x, y)$ par la formule, avec $\xi:=\left(1, \eta^{\prime}\right)$

$$
K(x, y):=\frac{1}{(2 \pi \sqrt{-1})^{n}} \int_{\widetilde{\beta}} d \eta^{\prime} \int_{\varrho}^{\infty} e^{-\tau(y-x) \cdot \xi} \tau^{n-1} P(x, \tau \xi) d \tau,
$$

$\varrho$ et $\widetilde{\beta}$ étant pris comme dans (4.7) et (4.8). K(x,y) est déterminé par $P(x, \xi)$ dans $\mathcal{O}_{X \times X}(V) / \sum_{k=1}^{n} \mathcal{O}_{X \times X}\left(V_{\widehat{k}}\right)$. Donc à tout symbole $P(x, \xi)$ correspond un opérateur pseudo-différentiel $P \in \mathcal{E}(\mathcal{G} ; D)$. En outre, $P=0$ si le symbole $P(x, \xi)$ appartient à $N^{\mathfrak{M}}(\Omega)$.

Maintenant on prouvera le théorème principal de cet article qui affirme que les correspondances définies dans les propositions 4.2 et 4.3 sont mutuellement réciproques : 
ThÉORÈme 4.4. Pour $K(x, y) d y \in \mathcal{O}_{X \times X}^{(0, n)}(V)$ et $P(x, \xi) \in S^{\mathfrak{M}}(\Omega)$, on a

$$
\begin{gathered}
\frac{1}{(2 \pi \sqrt{-1})^{n}} \int_{\widetilde{\beta}} d \eta^{\prime} \int_{\varrho}^{\infty} e^{-\tau(y-x) \cdot \eta} \tau^{n-1} d \tau \int_{\gamma} e^{\tau w \cdot \eta} K(x, x+w) d w \\
\equiv K(x, y) \quad \text { modulo } \sum_{k=1}^{n} \mathcal{O}_{X \times X}\left(V_{\widehat{k}}\right), \\
\frac{1}{(2 \pi \sqrt{-1})^{n}} \int_{\gamma} e^{z \cdot \xi} d z \int_{\widetilde{\beta}} d \eta^{\prime} \int_{\varrho}^{\infty} e^{-\tau z \cdot \eta} \tau^{n-1} P(x, \tau \eta) d \tau \\
\equiv P(x, \xi) \quad \text { modulo } N^{\mathfrak{M}}(\Omega)
\end{gathered}
$$

avec $\eta:=\left(1, \eta^{\prime}\right)$, où $D_{1}, D_{2}, \delta>0$ et $\Omega$ sont pris convenablement. Donc les correspondances (4.6) et (4.10) sont réciproques l'une à l'autre et, par conséquent, donnent l'isomorphisme entre $\mathcal{E}_{[p]}^{\mathbb{R}}$ et $\lim _{\longrightarrow} S^{\mathfrak{M}}(\Omega) / N^{\mathfrak{M}}(\Omega)$ où la limite inductive est prise en $\mathfrak{M}$ et $\Omega$ voisinage conique de $p$.

Démonstration. Nous prouvons d'abord (4.11). Dans la suite, on pose $z:=y-x$. Prenons $\gamma_{1}$ proche de $B_{1}(x)$. Pour $z$ tel que $\left(z_{1}-\gamma_{1}\right) \cap\left(\Gamma_{1}+\right.$ $M(x))=\emptyset$ et $z_{j}$ dans l'extérieur du domaine entouré par $\gamma_{j}(2 \leq j \leq n)$, en prenant $\varrho$ dans une direction un peu oscillée de 1 , on peut supposer que pour tout $\tau \in\left[\varrho, \varrho \infty\left[\right.\right.$ et tout $w_{1} \in \gamma_{1}$, on a $\operatorname{Re} \tau\left(z_{1}-w_{1}\right)>0$. Donc, dans la définition de $\widetilde{\beta}$, si l'on prend $\varepsilon>0$ bien petit, pour tous $\eta^{\prime} \in \widetilde{\beta}$ et $w \in \gamma$, on a $\operatorname{Re} \tau(z-w) \cdot \eta=\operatorname{Re} \tau\left(z_{1}-w_{1}\right)+\operatorname{Re} \tau\left(z^{\prime}-w^{\prime}\right) \cdot \eta^{\prime}>0$. Alors dans (4.11), on peut changer l'ordre d'intégration et par conséquent, le premier membre de (4.11) est égale à

$$
\frac{1}{(2 \pi \sqrt{-1})^{n}} \int_{\widetilde{\beta}} d \eta^{\prime} \int_{\gamma} K(x, x+w) d w \int_{\varrho}^{\infty} e^{-\tau(z-w) \cdot \eta} \tau^{n-1} d \tau .
$$

Par un calcul direct, on a alors

$$
\int_{\varrho}^{\infty} e^{-\tau(z-w) \cdot \eta} \tau^{n-1} d \tau=\frac{(n-1) !}{((z-w) \cdot \eta)^{n}}+O\left(((z-w) \cdot \eta)^{0}\right) .
$$

Maintenant pour tout $k=1, \ldots, n$, prenons de petits circuits $\gamma_{k}^{\circ} \subset \mathbb{C}$ autour de $z_{k}$ avec l'orientation positive et posons $\gamma^{\circ}:=\gamma_{1}^{\circ} \times \cdots \times \gamma_{n}^{\circ}$. Il est facile de vérifier que le

$$
\begin{aligned}
& \int_{\widetilde{\beta}} d \eta^{\prime} \int_{\gamma-(-1)^{n} \gamma^{\circ}} K(x, x+w) d w \int_{\varrho}^{\infty} e^{-\tau(z-w) \cdot \eta} \tau^{n-1} d \tau \equiv 0 \\
& \text { modulo } \sum_{k=1}^{n} \mathcal{O}_{X \times X}\left(V_{\widehat{k}}\right) .
\end{aligned}
$$

Parce que l'intégrale $\int_{\widetilde{\beta}} d \eta^{\prime} \int_{\gamma^{\circ}} d w=\int_{\widetilde{\beta}} d \eta^{\prime} \oint_{\gamma^{\circ}} d w$ correspondant à la partie 
holomorphe $O\left(((z-w) \cdot \eta)^{0}\right)$ est zéro, à $\sum_{k=1}^{n} \mathcal{O}_{X \times X}\left(V_{\widehat{k}}\right)$ près, (4.13) est égale à

$$
\begin{aligned}
\frac{(n-1) !}{(2 \pi \sqrt{-1})^{n}} \int_{\widetilde{\beta}} d \eta^{\prime} \oint_{\gamma^{\circ}} \frac{K(x, x+w)}{((w-z) \cdot \eta)^{n}} d w \\
=\frac{(n-1) !}{(2 \pi \sqrt{-1})^{n}} \oint_{\gamma^{\circ}} K(x, x+w) d w \int_{\widetilde{\beta}} \frac{d \eta^{\prime}}{((w-z) \cdot \eta)^{n}} \\
=\frac{(n-1) !}{(2 \pi \sqrt{-1})^{n}} \oint_{\gamma^{\circ}} K(x, x+w) d w \\
\quad \times \int_{0}^{z_{n}^{-1} \varepsilon} d \eta_{n} \cdots \int_{0}^{z_{2}^{-1} \varepsilon} \frac{d \eta_{2}}{\left(\left(w_{1}-z_{1}\right)+\left(w^{\prime}-z^{\prime}\right) \cdot \eta^{\prime}\right)^{n}} .
\end{aligned}
$$

Puisque, posant $z^{\prime \prime}:=\left(z_{3}, \ldots, z_{n}\right)$ etc., on a

$$
\begin{aligned}
& \int_{0}^{z_{2}^{-1} \varepsilon} \frac{1}{\left(\left(w_{1}-z_{1}\right)+\left(w^{\prime}-z^{\prime}\right) \cdot \eta^{\prime}\right)^{n}} d \eta_{2} \\
&=-\frac{1}{n-1} \frac{1}{w_{2}-z_{2}} \\
& \times\left[\frac{1}{\left(\left(w_{1}-z_{1}\right)+\frac{w_{2}-z_{2}}{z_{2}} \varepsilon+\left(w^{\prime \prime}-z^{\prime \prime}\right) \cdot \eta^{\prime \prime}\right)^{n-1}}\right. \\
&\left.-\frac{1}{\left(\left(w_{1}-z_{1}\right)+\left(w^{\prime \prime}-z^{\prime \prime}\right) \cdot \eta^{\prime \prime}\right)^{n-1}}\right]
\end{aligned}
$$

et le premier terme du seconde membre est holomorphe à $z_{1}=0$, (4.14) est égale, modulo $\sum_{k=1}^{n} \mathcal{O}_{X \times X}\left(V_{\widehat{k}}\right)$, à

$$
\begin{aligned}
\frac{(n-2) !}{(2 \pi \sqrt{-1})^{n}} \oint_{\gamma^{\circ}} \frac{K(x, x+w)}{w_{2}}-z_{2} d w \\
\quad \times \int_{0}^{z_{n}^{-1} \varepsilon} d \eta_{n} \cdots \int_{0}^{z_{3}^{-1} \varepsilon} \frac{d \eta_{3}}{\left(\left(w_{1}-z_{1}\right)+\left(w^{\prime}-z^{\prime}\right) \cdot \eta^{\prime}\right)^{n-1}} .
\end{aligned}
$$

En continuant ce procédé, (4.14) est égale, modulo $\sum_{k=1}^{n} \mathcal{O}_{X \times X}\left(V_{\widehat{k}}\right)$, à

$$
\frac{1}{(2 \pi \sqrt{-1})^{n}} \oint_{\gamma^{\circ}} \frac{K(x, x+w)}{\left(w_{2}-z_{2}\right) \cdots\left(w_{n}-z_{n}\right)} \frac{1}{w_{1}-z_{1}} d w \equiv K(x, x+z)=K(x, y),
$$

d'où (4.11).

Ensuite nous prouverons (4.12). Prenons $\gamma_{1}$ proche de $B_{1}(x)$ et un point $c \in M(x)$ tel que $H_{M(x)}\left(\xi_{0}\right)=\operatorname{Re}\left\langle c, \xi_{0}\right\rangle$. Alors il y a un point $d \in \gamma_{1}$ tel que 
$d-c_{1} \in \mathbb{R}_{+}$. On divise $\gamma_{1}$ en deux parties : $\gamma_{1}=\gamma_{1}^{+} \sqcup \gamma_{1}^{-}$avec

$$
\begin{aligned}
& \gamma_{1}^{+}:=\left\{z_{1} \in \gamma_{1} \mid \operatorname{Im}\left(z_{1}-c_{1}\right) \geq 0\right\}, \\
& \gamma_{1}^{-}:=\left\{z_{1} \in \gamma_{1} \mid \operatorname{Im}\left(z_{1}-c_{1}\right)<0\right\} .
\end{aligned}
$$

Si l'on prend $\delta>0$ assez petit, on peut prendre deux directions $\varrho_{+}$et $\varrho_{-}$ proches de la direction 1 telles qu'en posant

$$
\Sigma_{+}:=\left[\varrho_{+}, \varrho_{+} \infty\left[, \quad \Sigma_{-}:=\left[\varrho_{-}, \varrho_{-} \infty[,\right.\right.\right.
$$

on a $\operatorname{Re} z_{1} \cdot \tau>\operatorname{Re} c_{1} \cdot \tau=H_{M(x)}\left(\tau \xi_{0}\right)$ pour tout $z_{1} \in \gamma_{1}^{ \pm}$et $\tau \in \Sigma_{ \pm}$. On peut bien-entendu supposer que $\left|\varrho_{+}\right|=\left|\varrho_{-}\right|$et donc supposons $\varrho_{+}=r_{1} e^{\sqrt{-1} \theta_{+}}$ et $\varrho_{-}=r_{1} e^{\sqrt{-1} \theta_{-}}$avec $r_{1}>r$ et $-1 \ll \theta_{-}<0<\theta_{+} \ll 1$. Notons $\Sigma_{0}:=$ $\left\{r_{1} e^{\theta} \mid \theta_{-}<\theta<\theta_{+}\right\}$et $\Sigma:=\Sigma_{0} \sqcup \Sigma_{+} \sqcup \Sigma_{-}$. En posant $\gamma^{\prime}:=\gamma_{2} \times \cdots \times \gamma_{n}$, (4.12) est égale à

$$
\begin{aligned}
& \sum_{i= \pm} \frac{1}{(2 \pi \sqrt{-1})^{n}} \int_{\gamma_{1}^{i} \times \gamma^{\prime}} e^{z \cdot \xi} d z \int_{\widetilde{\beta}} d \eta^{\prime} \int_{\Sigma_{i}} e^{-\tau z \cdot \eta} \tau^{n-1} P(x, \tau \eta) d \tau \\
& =\frac{1}{(2 \pi \sqrt{-1})^{n}} \sum_{i= \pm \Sigma_{i}} \int_{\Sigma^{n}} \tau^{n-1} d \tau \oint_{\gamma^{\prime}} d z^{\prime} \int_{\widetilde{\beta}} P(x, \tau \eta) d \eta^{\prime} \int_{\gamma_{1}^{i}} e^{-z \cdot(\tau \eta-\xi)} d z_{1}
\end{aligned}
$$

et encore, à $N^{\mathfrak{M}}(\Omega)$ près, à

$$
\begin{aligned}
& \frac{1}{(2 \pi \sqrt{-1})^{n}} \sum_{i=0, \pm} \int_{\Sigma_{i}} \tau^{n-1} d \tau \oint_{\gamma^{\prime}} d z^{\prime} \int_{\widetilde{\beta}} P(x, \tau \eta) d \eta^{\prime} \int_{\gamma_{1}^{i}} e^{-z \cdot(\tau \eta-\xi)} d z_{1} \\
&=\frac{1}{(2 \pi \sqrt{-1})^{n}} \sum_{i=0, \pm \Sigma_{i}} \int^{n-1} d \tau \oint_{\gamma^{\prime}} d z^{\prime} \\
& \times \int_{\widetilde{\beta}} P(x, \tau \eta) e^{-z^{\prime} \cdot\left(\tau \eta^{\prime}-\xi^{\prime}\right)} d \eta^{\prime} \int_{\gamma_{1}^{i}} e^{-z_{1} \cdot\left(\tau-\xi_{1}\right)} d z_{1} .
\end{aligned}
$$

On a

$$
\begin{aligned}
\int_{\gamma_{1}^{-}} e^{-z_{1} \cdot\left(\tau-\xi_{1}\right)} d z_{1} & =\left[-\frac{1}{\tau-\xi_{1}} e^{-z_{1}\left(\tau-\xi_{1}\right)}\right]_{z_{1}=a}^{z_{1}=d} \\
& =\frac{1}{\tau-\xi_{1}} e^{-a\left(\tau-\xi_{1}\right)}-\frac{1}{\tau-\xi_{1}} e^{-d\left(\tau-\xi_{1}\right)}
\end{aligned}
$$

et

$$
\int_{\Sigma_{i}} d \tau \oint_{\gamma^{\prime}} d z^{\prime} \int_{\widetilde{\beta}} \frac{P(x, \tau \eta)}{\tau-\xi_{1}} \tau^{n-1} e^{-a\left(\tau-\xi_{1}\right)-z^{\prime} \cdot\left(\tau \eta^{\prime}-\xi^{\prime}\right)} d \eta^{\prime}
$$

appartient à $N^{\mathfrak{M}}(\Omega)$, et de même pour $i=+$. Donc par la formule de Cauchy à poids, modulo $N^{\mathfrak{M}}(\Omega)$, (4.16) est égale à 


$$
\begin{aligned}
\frac{1}{(2 \pi \sqrt{-1})^{n}} \oint_{\gamma^{\prime}} e^{z^{\prime} \cdot \xi^{\prime}} d z^{\prime} \int_{\widetilde{\beta}} d \eta^{\prime} \\
\quad \times \int_{\Sigma_{+}-\Sigma_{0}-\Sigma_{-}} \frac{P(x, \tau \eta) \tau^{n-1}}{\tau-\xi_{1}} e^{-d\left(\tau-\xi_{1}\right)} e^{-\tau z^{\prime} \cdot \eta^{\prime}} d \tau \\
=\frac{1}{(2 \pi \sqrt{-1})^{n}} \oint_{\gamma^{\prime}} e^{z^{\prime} \cdot \xi^{\prime}} d z^{\prime} \int_{\widetilde{\beta}}(2 \pi \sqrt{-1}) P\left(x, \xi_{1} \eta\right) \xi_{1}^{n-1} e^{-\xi_{1} z^{\prime} \cdot \eta^{\prime}} d \eta^{\prime} \\
=\frac{\xi_{1}^{n-1}}{(2 \pi \sqrt{-1})^{n-1}} \oint_{\gamma^{\prime}} d z^{\prime} \int_{\widetilde{\beta}} P\left(x, \xi_{1} \eta\right) e^{-z^{\prime} \cdot\left(\xi_{1} \eta^{\prime}-\xi^{\prime}\right)} d \eta^{\prime} \\
=\frac{\xi_{1}^{n-1}}{(2 \pi \sqrt{-1})^{n-1}} \oint_{\gamma_{n}} d z_{n} \int_{0}^{z_{n}^{-1} \varepsilon} e^{-z_{n}\left(\xi_{1} \eta_{n}-\xi_{n}\right)} d \eta_{n} \cdots \\
\quad \times \oint_{\gamma_{2} d z_{2}} \int_{0}^{-1} \varepsilon e^{-z_{2}\left(\xi_{1} \eta_{2}-\xi_{2}\right)} P\left(x, \xi_{1} \eta\right) d \eta_{2} \cdot
\end{aligned}
$$

Puisque $P(x, \xi)$ est holomorphe sur le voisinage conique $\Omega(r)$ de $p=\left(x_{0}, \xi_{0}\right)$ et $\eta:=(1,0, \ldots, 0)$, en prenant $\varepsilon>0$ si petit que $0<\left|z_{j} \eta_{j}\right| \ll 1$ (pour $2 \leq j \leq n)$, la fonction $P\left(x, \xi_{1} \eta\right)=P\left(x ; \xi_{1}, \xi_{1} \eta_{2}, \ldots, \xi_{1} \eta_{n}\right)$ est holomorphe en $\eta^{\prime}=0$. Alors en posant $\eta^{\prime \prime}:=\left(\eta_{3}, \ldots, \eta_{n}\right)$, (4.18) est égale à

$$
\begin{aligned}
& \left(\frac{\xi_{1}}{2 \pi \sqrt{-1}} \oint_{\gamma_{n}} e^{z_{n} \xi_{n}} d z_{n} \int_{0}^{z_{n}^{-1} \varepsilon} e^{-z_{n} \xi_{1} \eta_{n}} d \eta_{n}\right) \times \cdots \\
& \times\left(\frac{\xi_{1}}{2 \pi \sqrt{-1}} \oint_{\gamma_{2}} e^{z_{2} \xi_{2}} d z_{2} \int_{0}^{z_{2}^{-1} \varepsilon} e^{-z_{2} \xi_{1} \eta_{2}} P\left(x, \xi_{1}, \xi_{1} \eta_{2}, \xi_{1} \eta^{\prime \prime}\right) d \eta_{2}\right) .
\end{aligned}
$$

Le changement de variable $\lambda:=z_{2} \xi_{1} \eta_{2}$ donne

$$
\begin{aligned}
\frac{\xi_{1}}{2 \pi \sqrt{-1}} \oint_{\gamma_{2}} e^{z_{2} \xi_{2}} d z_{2} & \int_{0}^{z_{2}^{-1} \varepsilon} e^{-z_{2} \xi_{1} \eta_{2}} P\left(x, \xi_{1}, \xi_{1} \eta_{2}, \xi_{1} \eta^{\prime \prime}\right) d \eta_{2} \\
& =\frac{1}{2 \pi \sqrt{-1}} \oint_{\gamma_{2}} e^{z_{2} \xi_{2}} d z_{2} \int_{0}^{\xi_{1} \varepsilon} \frac{e^{-\lambda}}{z_{2}} P\left(x, \xi_{1}, \frac{\lambda}{z_{2}}, \xi_{1} \eta^{\prime \prime}\right) d \lambda \\
& =\frac{1}{2 \pi \sqrt{-1}} \int_{0}^{\xi_{1} \varepsilon} e^{-\lambda} d \lambda \oint_{\gamma_{2}} \frac{P\left(x, \xi_{1}, \frac{\lambda}{z_{2}}, \xi_{1} \eta^{\prime \prime}\right)}{z_{2}} e^{z_{2} \xi_{2}} d z_{2}
\end{aligned}
$$

Si l'on fait le changement de variable $w_{2}:=1 / z_{2}$ et on munit le cercle $\widetilde{\gamma}_{2}:=$ $\left\{w_{2} \mid 1 / w_{2} \in \gamma_{2}\right\}$ de la même orientation que $\gamma_{2}$, cela est égale à 


$$
\begin{aligned}
\frac{1}{2 \pi \sqrt{-1}} \int_{0}^{\xi_{1} \varepsilon} e^{-\lambda} & d \lambda \oint_{\widetilde{\gamma}_{2}} \frac{P\left(x, \xi_{1}, \lambda w_{2}, \xi_{1} \eta^{\prime \prime}\right)}{w_{2}} \sum_{i=0}^{\infty} \frac{\xi_{2}^{i}}{i !} w_{2}^{-i} d w_{2} \\
& =\frac{1}{2 \pi \sqrt{-1}} \sum_{i=0}^{\infty} \frac{\xi_{2}^{i}}{i !} \int_{0}^{\xi_{1} \varepsilon} e^{-\lambda} d \lambda \oint_{\widetilde{\gamma}_{2}} \frac{P\left(x, \xi_{1}, \lambda w_{2}, \xi_{1} \eta^{\prime \prime}\right)}{w_{2}^{i+1}} d w_{2} \\
& =\sum_{i=0}^{\infty} \int_{0}^{\xi_{1} \varepsilon} e^{-\lambda} \frac{\xi_{2}^{i}}{(i !)^{2}} \lambda^{i} \partial_{\xi_{2}}^{i} P\left(x, \xi_{1}, 0, \xi_{1} \eta^{\prime \prime}\right) d \lambda \\
& =\sum_{i=0}^{\infty} \frac{\partial_{\xi_{2}}^{i} P\left(x, \xi_{1}, 0, \xi_{1} \eta^{\prime \prime}\right)}{(i !)^{2}} \xi_{2}^{i} \int_{0}^{\xi_{1} \varepsilon} \lambda^{i} e^{-\lambda} d \lambda
\end{aligned}
$$

Puisque $\int_{0}^{\xi_{1} \varepsilon} \lambda^{i} e^{-\lambda} d \lambda \equiv i$ ! modulo $N^{\mathfrak{M}}(\Omega)$, ce dernier est égale à

$$
\sum_{i=0}^{\infty} \frac{\partial_{\xi_{2}}^{i} P\left(x, \xi_{1}, 0, \xi_{1} \eta^{\prime \prime}\right)}{i !} \xi_{2}^{i}=P\left(x, \xi_{1}, \xi_{2}, \xi_{1} \eta^{\prime \prime}\right)
$$

En répétant ce procédé, finalement (4.19) est égale à $P(x, \xi)$ à $N^{\mathfrak{M}}(\Omega)$ près, et ceci enfin prouve (4.12).

Pour finir cette section, on récrit la composition au langage des symboles. Soient $\Gamma_{1}, \ldots, \Gamma_{n}$ et $\Gamma_{1}^{\prime}, \ldots, \Gamma_{n}^{\prime}$ deux classes de cônes de la forme (3.4)-(3.5) pour $\delta>0$ et $\delta^{\prime}>0$, et $\mathfrak{M}=(M(x)), \mathfrak{M}^{\prime}=\left(M^{\prime}(x)\right)$ deux familles de compacts convexes. Définissons aussi $\mathcal{G}$ et $\mathcal{G}^{\prime}$ correspondant respectivement à $\Gamma_{1}, \ldots, \Gamma_{n}$ et $\Gamma_{1}^{\prime}, \ldots, \Gamma_{n}^{\prime}$ et à $\mathfrak{M}, \mathfrak{M}^{\prime}$. Considérons $Z:=Z_{\mathcal{G}}$ et $Z^{\prime}:=Z_{\mathcal{G}^{\prime}}$ et posons $\mathfrak{M}^{\prime \prime}=\mathfrak{M}+\mathfrak{M}^{\prime}:=\left(M(x)+M^{\prime}(x)\right)$. Pour $\delta^{\prime \prime}>0$ avec $0<\delta^{\prime \prime}<\delta^{\prime}$, à partir de $\Gamma_{1}^{\prime \prime}, \ldots, \Gamma_{n}^{\prime \prime}$ définies comme dans $(3.4)-(3.5)$ et $\mathfrak{M}^{\prime \prime}$, on définit $\mathcal{G}^{\prime \prime}$ et on pose $Z^{\prime \prime}:=Z_{\mathcal{G}}^{\prime \prime}$. Alors on a $Z^{\prime \prime} \supset Z^{\prime} \circ Z$ et $Z^{\prime \prime}$ est un $\mathfrak{M}^{\prime \prime}$-ordinal tel que $q_{13}: q_{1,2}^{-1} Z \cap q_{2,3}^{-1} Z^{\prime} \rightarrow Z^{\prime \prime}$ soit propre. Alors pour tous ouverts $D_{1}, D_{2}, D_{1}^{\prime}, D_{2}^{\prime}$ qui sont $\left(Z, Z^{\prime}\right)$-ronds, la composition de la proposition 3.6 donne une application $\mathcal{E}\left(\mathcal{G} ; D_{1}, D_{2}\right) \otimes \mathcal{E}\left(\mathcal{G}^{\prime} ; D_{1}^{\prime}, D_{2}^{\prime}\right) \rightarrow \mathcal{E}\left(\mathcal{G}^{\prime \prime} ; D_{1}, D_{2}^{\prime}\right)$. Comme exactement dans la proposition 4.4 de [9], on a

Proposition 4.5 (La formule d'Hörmander-Leibniz). Dans la situation ci-dessus, pour tout $P \in \mathcal{E}\left(\mathcal{G}^{\prime} ; D_{1}^{\prime}, D_{2}^{\prime}\right)$ et tout $Q \in \mathcal{E}\left(\mathcal{G} ; D_{1} . D_{2}\right)$, il existe des symboles respectifs $\sigma(P)(x, \xi)=P(x, \xi) \in S^{\mathfrak{M}^{\prime}}\left(\Omega^{\prime}\right)$ et $\sigma(Q)(x, \xi)=$ $Q(x, \xi) \in S^{\mathfrak{M}}(\Omega), \Omega^{\prime}, \Omega$ étant comme au début de la section, tels que l'on ait la formule suivante dont le second membre appartient à $S_{1}^{\mathfrak{M}^{\prime \prime}}\left(\Omega \cap \Omega^{\prime}\right)$ et converge compactement dans $\Omega \cap \Omega^{\prime}$ :

$$
\sigma(Q \circ P)=\sum_{\alpha \in \mathbb{Z}_{+}^{n}} \frac{1}{\alpha !} \partial_{\xi}^{\alpha} Q(x, \xi) \cdot \partial_{x}^{\alpha} P(x, \xi) .
$$


Démonstration. On peut supposer $\Omega=\Omega^{\prime}$, sinon on prends $\Omega, \Omega^{\prime}$ plus petits. Soient $L(x, y) d y \in \mathcal{O}_{X \times X}^{(0, n)}\left(V^{\prime}\right)$ et $K(x, y) d y \in \mathcal{O}_{X \times X}^{(0, n)}(V)$, respectivement, des noyaux de $P$ et $Q$. On peut prendre un chemin d'intégration $\lambda$ de même type que $\gamma$. Alors on a, à $N^{\mathfrak{M}^{\prime \prime}}(\Omega)$ près,

$$
\begin{aligned}
\sigma(Q \circ P)(x, \xi) & =\int_{\gamma} e^{z \cdot \xi} d z \int_{\lambda} L(x, w) K(w, x+z) d w \\
& =\int_{\lambda} L(x, w) d w \int_{\gamma+(x-w)} e^{(u+w-x) \cdot \xi} K(w, w+u) d u \\
& \equiv \int_{\lambda} e^{(w-x) \cdot \xi} L(x, w) d w \int_{\gamma} e^{u \cdot \xi} K(w, w+u) d u \\
& =\int_{\lambda} e^{v \cdot \xi} L(x, x+v) P(x+v, \xi) d v \\
& =\sum_{\alpha \geq 0} \frac{1}{\alpha !} \partial_{x}^{\alpha} P(x, \xi) \int_{\lambda} e^{v \cdot \xi} v^{\alpha} L(x, x+v) d v \\
& \equiv \sum_{\alpha \geq 0} \frac{1}{\alpha !} \partial_{x}^{\alpha} P(x, \xi) \int_{\lambda} e^{v \cdot \xi} v^{\alpha} L(x, x+v) d v \\
& =\sum_{\alpha \geq 0} \frac{1}{\alpha !} \partial_{x}^{\alpha} P(x, \xi) \partial_{\xi}^{\alpha} \int_{\lambda}^{v \cdot \xi} L(x, x+v) d v \\
& =\sum_{\alpha \in \mathbb{Z}_{+}^{n}} \frac{1}{\alpha !} \partial_{\xi}^{\alpha} Q(x, \xi) \cdot \partial_{x}^{\alpha} P(x, \xi) .
\end{aligned}
$$

5. Les symboles formels. Dans cette section, on pose $\mathbb{Z}_{+}:=\{\nu \in \mathbb{Z} \mid$ $\nu \geq 0\}$. Nous introduisons les symboles formels :

DÉfinition 5.1. Pour une famille de supports $\mathfrak{M}:=(M(x))$ et un voisinage conique $\Omega$ de $p=\left(x_{0}, \xi_{0}\right)$, on définit

$$
\begin{aligned}
\widehat{S}^{\mathfrak{M}}(\Omega):=\{P( & t ; x, \xi)=\sum_{\nu=0}^{\infty} t^{\nu} P_{\nu}(x, \xi) \mid \text { il existe } r>0 \text { tel que } \\
& P_{\nu}(x, \xi) \in \mathcal{O}(\Omega((\nu+1) r))\left(\forall \nu \in \mathbb{Z}_{+}\right) \text {et on ait : } \\
& \text { pour tout ensemble conique } \Omega^{\prime} \Subset \Omega, \text { il existe } d>r \\
& \text { et } A>0 \text { tels que } 0<A<1 \text { et pour tout } \varepsilon>0, \\
& \text { avec } C_{\varepsilon}>0 \text { on ait }\left|P_{\nu}(x, \xi)\right| \leq C_{\varepsilon} A^{\nu} e^{H_{M(x)}(\xi)+\varepsilon|\xi|} \\
& \text { (pour tous } \left.\left.\nu \geq 0 \text { et }(x, \xi) \in \Omega^{\prime}[(\nu+1) d]\right)\right\}
\end{aligned}
$$

et $\widehat{N}^{\mathfrak{M}}(\Omega)$ est le sous-ensemble des $P(t ; x, \xi)=\sum_{\nu=0}^{\infty} t^{\nu} P_{\nu}(x, \xi) \in \widehat{S}^{M}(\Omega)$ tel que $P_{\nu}(x, \xi) \in \mathcal{O}(\Omega((\nu+1) r))$, pour tout $q=(y, \eta) \in \Omega(r)$, il existe $d>r, A>0$ et un voisinage conique $\Omega^{\prime} \Subset \Omega$ de $q$ tels que $0<A<1$ et 


$$
0 \leq \mu=\mu_{\Omega^{\prime}}:=\max _{(x, \xi) \in \Omega^{\prime}, \xi \neq 0}\left[H_{M(x)}(\xi /|\xi|)-I_{M(x)}(\xi /|\xi|)\right]<-\frac{\ln A}{d},
$$

et pour tout $\varepsilon>0$, avec $C_{\varepsilon}>0$ on ait

$$
\left|\sum_{\nu=0}^{m-1} P_{\nu}(x, \xi)\right| \leq C_{\varepsilon} A^{m} e^{H_{M(x)}(\xi)+\varepsilon|\xi|} \quad \forall m \geq 1, \forall(x, \xi) \in \Omega^{\prime}[m d] .
$$

Appelons tout $P(t ; x, \xi) \in \widehat{S}^{\mathfrak{M}}(\Omega)$ un symbole formel et tout $Q(t ; x, \xi) \in$ $\widehat{N}^{\mathfrak{M}}(\Omega)$ un symbole formel $n u l$.

Par le même raisonnement que dans [9] (ou plutôt originellement par le raisonnement de Aoki [2]), on peut démontrer qu'un opérateur pseudodifférentiel est représenté, aux symboles formels nuls près, par un symbole formel. Pour cela, on a besoin de quelques propositions. D'abord on a évidemment :

Proposition 5.2. Le morphisme suivant est une injection:

$$
P(x, \xi) \mapsto\left[P(x, \xi)+0 t+0 t^{2}+\cdots\right]: S^{\mathfrak{M}}(\Omega) \hookrightarrow \widehat{S}^{\mathfrak{M}}(\Omega) .
$$

Proposition 5.3. On a l'égalité

$$
S^{\mathfrak{M}}(\Omega) \cap \widehat{N}^{\mathfrak{M}}(\Omega)=N^{\mathfrak{M}}(\Omega) .
$$

Démonstration. Pour tout $P(x, \xi) \in S^{\mathfrak{M}}(\Omega) \cap \widehat{N}^{\mathfrak{M}}(\Omega)$, d'après la définition 5.1, pour tout $q \in \Omega(r)$, il existe un voisinage conique $\Omega^{\prime} \Subset \Omega$ de $q$, $d>r$ et $A>0$ avec $0<A<1$ tels que $\mu<-(\ln A) / d$ et que pour tout $\varepsilon>0$, il y ait $C_{\varepsilon}>0$ et on ait

$$
|P(x, \xi)| \leq C_{\varepsilon} A^{m} e^{H_{M(x)}(\xi)+\varepsilon|\xi|} \quad \forall m \geq 1, \forall(x, \xi) \in \Omega^{\prime}[m d] .
$$

En prenant $\delta>0$ assez petit, on a $\varepsilon_{0}:=-(\delta+(\ln A) / d+\mu)>0$. Pour tout $(x, \xi) \in \Omega^{\prime}[m d]$, il existe $m \geq 1$ tel que $d(m+1) \geq|\xi| \geq m d$, donc on a facilement

$$
|P(x, \xi)| \leq C_{\delta} A^{-1} \exp \left(I_{M(x)}(\xi)-\varepsilon_{0}\right) .
$$

Puisque $q \in \Omega(r)$ est quelconque, il s'ensuit que $P(x, \xi) \in N^{\mathfrak{M}}(\Omega)$.

Réciproquement, pour tout $P(x, \xi) \in N^{\mathfrak{M}}(\Omega)$, d'après la définition 4.1, pour tous $\Omega^{\prime} \Subset \Omega$ et $d>r$ avec $d \geq 1$, il existe $\varepsilon_{0}, C>0$ tels que $\varepsilon_{0}<1$ et

$$
|P(x, \xi)| \leq C e^{I_{M}(\xi)-\varepsilon_{0}|\xi|} \quad \text { sur } \Omega^{\prime}[d] .
$$

Pour tous $\varepsilon_{1}>0$ avec $0<\varepsilon_{1}<\varepsilon_{0}$ et $q \in \Omega(r)$, il existe un voisinage conique $\Omega^{\prime} \Subset \Omega$ de $q$ tel que $I_{M(x)}(\xi) \leq H_{M(x)}(\xi)-\mu|\xi|+\varepsilon_{1}|\xi|$ sur $\Omega^{\prime}$. Alors $A:=e^{-d\left(\varepsilon_{0}-\varepsilon_{1}+\mu\right)}<1$ satisfait à la condition (5.2) et pour tout $(x, \xi) \in \Omega^{\prime}[m d]$, on a facilement

$$
|P(x, \xi)| \leq C A^{m} e^{H_{M}(\xi)},
$$

ce qui prouve $P(x, \xi) \in \widehat{N}^{\mathfrak{M}}(\Omega)$. 
ThÉORÈme 5.4. Pour tout symbole formel $P(t ; x, \xi)=\sum_{\nu=0}^{\infty} t^{\nu} P_{\nu}(x, \xi)$ $\in \widehat{S}^{\mathfrak{M}}(\Omega)$, il existe un voisinage conique ouvert $\Omega^{\prime} \Subset \Omega$ de $p$ et un symbole $P(x, \xi) \in S^{\mathfrak{M}}\left(\Omega^{\prime}\right)$ tels que

$$
P(t ; x, \xi)-P(x, \xi) \in \widehat{N}^{\mathfrak{M}}\left(\Omega^{\prime}\right) .
$$

En particulier, compte tenu des propositions 5.2 et 5.3 , on a l'isomorphisme

$$
\iota: S^{\mathfrak{M}}(\Omega) / N^{\mathfrak{M}}(\Omega) \stackrel{\sim}{\rightarrow} \widehat{S}^{\mathfrak{M}}(\Omega) / \widehat{N}^{\mathfrak{M}}(\Omega) .
$$

Démonstration. La démonstration est analogue à celle de la proposition 6.7 de [9] et donc nous la ferons brièvement. Dans la situation de la proposition 4.3 et la définition 5.1 , pour tout $\nu$, posons

$$
K_{\nu}(x, y):=\frac{1}{(2 \pi \sqrt{-1})^{n}} \int_{\widetilde{\beta}} d \eta^{\prime} \int_{(\nu+2) d}^{\infty} e^{-\tau(y-x) \cdot \xi} \tau^{n-1} P_{\nu}(x, \tau \xi) d \tau
$$

où $\widetilde{\beta}$ vient de $(4.8)$ et

$$
\widetilde{P}_{\nu}(x, \xi):=\int_{\gamma} e^{z \cdot \xi} K_{\nu}(x, x+z) d z
$$

où $\gamma$ est comme dans (4.6). D'après les propositions 4.3 et 4.2 , il existe un voisinage conique ouvert $\Omega^{\prime} \Subset \Omega$ de $p$, indépendant de $\nu$, tel que $\widetilde{P}_{\nu}(x, \xi) \in$ $S^{\mathfrak{M}}\left(\Omega^{\prime}\right)$ et $\widetilde{P}_{\nu}(x, \xi) \in \mathcal{O}\left(\Omega^{\prime}[r]\right)$ pour tout $r>0$. Alors on voit facilement que la série $\sum_{\nu=0}^{\infty} \widetilde{P}_{\nu}(x, \xi)$ converge absolument et compactement sur $\Omega^{\prime}[r]$ et définit un symbole $P(x, \xi) \in S^{\mathfrak{M}}\left(\Omega^{\prime}\right)$ et que

$$
P(x, \xi)-\sum_{\nu=0}^{\infty} t^{\nu} \widetilde{P}_{\nu}(x, \xi) \in \widehat{N}^{\mathfrak{M}}\left(\Omega^{\prime}\right)
$$

D'après le théorème 4.4 , on a

$$
\sum_{\nu=0}^{\infty} t^{\nu} P_{\nu}(x, \xi)-\sum_{\nu=0}^{\infty} t^{\nu} \widetilde{P}_{\nu}(x, \xi) \in \widehat{N}^{\mathfrak{M}}\left(\Omega^{\prime}\right)
$$

d'où

$$
P(x, \xi)-\sum_{\nu=0}^{\infty} t^{\nu} P_{\nu}(x, \xi) \in \widehat{N}^{\mathfrak{M}}\left(\Omega^{\prime}\right) .
$$

Par le même raisonnement que dans [9, Proposition 6.10], on peut aussi démontrer :

Proposition 5.5 (Leibniz-Hörmander). Soient $\mathfrak{M}, \mathfrak{M}^{\prime} \subset X$ deux familles de supports satisfaisant à (4.1) et $\Omega, \Omega^{\prime}$ deux voisinages coniques ouverts de $p$. Pour tous symboles $P(t ; x, \xi) \in \widehat{S}^{\mathfrak{M}^{\prime}}\left(\Omega^{\prime}\right)$ et $Q(t ; x, \xi) \in \widehat{S}^{\mathfrak{M}}(\Omega)$, $P, Q$ étant les opérateurs pseudo-différentiels correspondants, le symbole 
formel de la composition $\sigma(Q \circ P) \in \widehat{S}^{\mathfrak{M}^{\prime \prime}}\left(\Omega \cap \Omega^{\prime}\right)$ est calculé par

$$
\left.e^{t \partial_{\xi} \cdot \partial_{y}} Q(t ; x, \xi) P(t ; y, \eta)\right|_{y=x, \eta=\xi}:=\sum_{\alpha \in \mathbb{Z}_{+}^{n}} \frac{t^{|\alpha|}}{\alpha !} \partial_{\xi}^{\alpha} Q(t ; x, \xi) \partial_{x}^{\alpha} P(t ; x, \xi)
$$

où $\mathfrak{M}^{\prime \prime}$ a été défini avant la proposition 4.5.

\section{Théorème d'inversibilité pour les opérateurs pseudo-différen-} tiels en un point. Dans cette section, comme Aoki l'a fait pour le cas des opérateurs pseudo-différentiels locaux (voir [3]), on développera le calcul exponentiel pour le symbole formel et en utilisant ce calcul, on établira le théorème d'inversibilité pour quelques opérateurs pseudo-différentiels définis en un point. Dans cette section, on suppose que $x_{0}=0$ et que la famille $\mathfrak{M}=(M(x))$ satisfait à la condition suivante qui est plus forte que l'hypothèse $(4.1)$ :

$$
\max _{|\xi|=1}\left|H_{M(x)}(\xi)\right|=o(|x|) \quad(|x| \rightarrow 0) .
$$

Dans la suite, pour tout $x \in X$, on écrira aussi pour la simplicité $H_{x}=$ $\max _{|\xi|=1}\left|H_{M(x)}(\xi)\right|$.

DÉfinition 6.1. Soit $\mathfrak{M}$ une famille de supports et soit 0 la famille de supports consistant de l'ensemble $\{0\}$. Un symbole formel $p(t ; x, \xi)=$ $\sum_{j=0}^{\infty} t^{j} p_{j}(x, \xi) \in \widehat{S}^{0}(\Omega)$ satisfaisant à (5.2) est dit du type $\mathfrak{M}^{+}$(resp. $\mathfrak{M}^{-}$) dans $\Omega$ s'il satisfait en outre à la condition suivante : pour tout ensemble conique $\Omega^{\prime} \Subset \Omega$ et tout $\delta>0$, il existe $d>r$ tel que pour tout $\varepsilon>0$, il y ait $C_{\varepsilon}>0$ tel que

$$
\begin{aligned}
& \operatorname{Re} p_{0}(x, \xi) \leq H_{M(x)}(\xi)+\varepsilon|\xi|+C_{\varepsilon} \quad\left(\text { resp. } \leq I_{M(x)}(\xi)+\varepsilon|\xi|+C_{\varepsilon}\right), \\
& \left|\operatorname{Im} p_{0}(x, \xi)\right| \leq \varepsilon|\xi|+C_{\varepsilon} \quad \operatorname{sur} \Omega^{\prime}[d], \\
& \left|p_{j}(x, \xi)\right| \leq \delta^{j}\left(H_{x} \cdot|\xi|+\varepsilon|\xi|+C_{\varepsilon}\right) \quad \text { sur } \Omega^{\prime}[(j+1) d](j=1,2, \ldots) .
\end{aligned}
$$

Si l'on prend $\varepsilon>0$ assez petit, (6.4) se remplace aussi par

$$
\left|p_{j}(x, \xi)\right| \leq \delta^{j}\left(H_{M(x)}(\xi)+C_{\varepsilon}\right)
$$

$\mathrm{Ou}$

$$
\left|p_{j}(x, \xi)\right| \leq \delta^{j}\left(\varepsilon|\xi|+C_{\varepsilon}\right)
$$

$\operatorname{sur} \Omega[(j+1) d]$ pour $j \geq 1$.

Proposition 6.2. Si $p(t ; x, \xi) \in \widehat{S}^{0}(\Omega)$ est du type $\mathfrak{M}^{+}$, alors le symbole $P(t ; x, \xi):=e^{p(t ; x, \xi)}$, calculé formellement, appartient $\grave{a} \widehat{S}^{\mathfrak{M}}(\Omega)$. 
Démonstration. Suppposons que $p(t ; x, \xi)=\sum_{j=0}^{\infty} t^{j} p_{j}(x, \xi)$ satisfait à (6.2)-(6.4). Alors, formellement, le second membre est donné par

$$
e^{p(t ; x, \xi)}=1+\sum_{k=1}^{\infty} \frac{1}{k !} \sum_{j_{1}, \ldots, j_{k}=0}^{\infty} t^{j_{1}+\cdots+j_{k}} p_{j_{1}}(x, \xi) \cdots p_{j_{k}}(x, \xi) .
$$

Donc la fait que cela est égale à $P(t ; x, \xi) \equiv \sum_{\nu=0}^{\infty} t^{\nu} P_{\nu}(x, \xi)$ veut dire que pour tout $\nu \in \mathbb{Z}_{+}$, on a

$$
P_{\nu}(x, \xi)= \begin{cases}1+\sum_{k=1}^{\infty} \frac{1}{k !} p_{0}(x, \xi)^{k}=e^{p_{0}(x, \xi)} & (\nu=0), \\ \sum_{k=1}^{\infty} \frac{1}{k !} \sum_{j_{1}+\cdots+j_{k}=\nu} p_{j_{1}}(x, \xi) \cdots p_{j_{k}}(x, \xi) & (\nu \geq 1) .\end{cases}
$$

D'après (6.2)-(6.4), on a aisément $P(t ; x, \xi)=\sum_{\nu=0}^{\infty} t^{\nu} P_{\nu}(x, \xi) \in \widehat{S}^{\mathfrak{M}}(\Omega)$, comme dans la preuve de la proposition 3.2 de [10].

Proposition 6.3. Supposons que la famille de supports $\mathfrak{M}=(M(x))$ satisfait à (6.1) et que $H_{c x} \leq c H_{x}$ pour tout $c>0$. Soient les symboles $p(x, \xi) \in S^{0}(\Omega), r(t ; x, \xi) \in \widehat{S}^{0}(\Omega)$ respectivement du type $\mathfrak{M}^{+}, 0^{+}$et soit $q(t ; x, \xi) \in \widehat{S}^{0}(\Omega)$. Dans la situation de la définition 6.1 , supposons en outre que pour tout conique $\Omega^{\prime} \Subset \Omega$, il existe $d^{\prime}>d$ tel que pour tout $\varepsilon>0$, il existe $C>0$ tel que

$$
\left\{\begin{array}{l}
\operatorname{Re} p(x, \xi) \geq H_{M(x)}(\xi)-\varepsilon|\xi|-C, \\
\operatorname{Re} r_{0}(x, \xi) \geq-\varepsilon|\xi|-C
\end{array}\right.
$$

sur $\Omega^{\prime}\left[d^{\prime}\right]$. Posons $P(x, \xi):=e^{p(x, \xi)}, Q(t ; x, \xi):=e^{q(t ; x, \xi)}, R(t ; x, \xi):=$ $e^{r(t ; x, \xi)}$ et désignons par $P, Q, R$ respectivement les opérateurs correspondants. Si en outre, on a formellement $\sigma(P \circ Q)=\sigma(R)$, alors il existe un voisinage conique $\Omega^{\prime} \subset \Omega$ de $p$ tel que $q(t ; x, \xi)$ soit du type $(-\mathfrak{M})^{-}$sur $\Omega^{\prime}$, où $-\mathfrak{M}:=(-M(x))$ si $\mathfrak{M}=(M(x))$.

Démonstration. Cette proposition se démontre aussi comme la proposition 3.4 de [10]. D'après la proposition 5.5, le symbole de la composition $\sigma(P \circ Q)$ sera calculé comme suit : posons formellement

$$
W(s, t ; x, y, \xi, \eta):=e^{s \partial_{\xi} \cdot \partial_{y}} e^{p(x, \xi)+q(t ; y, \eta)} ;
$$

alors on a formellement $\sigma(P \circ Q)(t ; x, \xi)=W(t, t ; x, x, \xi, \xi)$. Par (6.8), toujours formellement, $W$ satisfait à

$$
\left\{\begin{array}{l}
\partial_{s} W=\left(\partial_{\xi} \cdot \partial_{y}\right) W \\
W(0, t ; x, y, \xi, \eta)=e^{p(t ; x, \xi)+q(t ; y, \eta)}
\end{array}\right.
$$

Si $W$ est de la forme

$$
W(s, t ; x, y, \xi, \eta)=e^{\sum_{k=0}^{\infty} s^{k} v_{k}(x, y, \xi, \eta)},
$$


en posant $w_{k}(s, t ; x, y, \xi, \eta):=s^{k} v_{k}(x, y, \xi, \eta),(6.9)$ revient à dire que, pour $k \geq 0$,

$(6.10)_{0}$

$$
\begin{aligned}
& w_{0}(s, t ; x, y, \xi, \eta)=p(t ; x, \xi)+q(t ; y, \eta) \\
& w_{k+1}(s, t ; x, y, \xi, \eta)=\frac{s}{k+1}\left(\left(\partial_{\xi} \cdot \partial_{y}\right) w_{k}(s, t ; x, y, \xi, \eta)\right. \\
&\left.+\sum_{\nu=0}^{k} \partial_{\xi} w_{\nu}(s, t ; x, y, \xi, \eta) \cdot \partial_{y} w_{k-\nu}(s, t ; x, y, \xi, \eta)\right) .
\end{aligned}
$$$$
(6.10)_{k+1}
$$

L'hypothèse de la proposition est

$$
r(t ; x, \xi)=\sum_{k=0}^{\infty} w_{k}(t, t ; x, x, \xi, \xi) .
$$

En développant

$$
q(t ; x, \xi)=\sum_{j=0}^{\infty} t^{j} q_{j}(x, \xi), \quad v_{k}(t ; x, y, \xi, \eta)=\sum_{l=0}^{\infty} t^{l} w_{k}^{l}(x, y, \xi, \eta),
$$

$(6.10)_{0}$ est interprété comme

$$
w_{0}(t, t ; x, y, \xi, \eta)=\sum_{l=0}^{\infty} t^{l} w_{0}^{l}(x, y, \xi, \eta)=p(x, \xi)+\sum_{j=0}^{\infty} t^{j} q_{j}(y, \eta) .
$$

Donc d'après $(6.11)$, pour tout $j \geq 1$ on a

$$
r_{j}(x, \xi)=\sum_{k=0}^{j} w_{k}^{j-k}(x, x, \xi, \xi)=q_{j}(x, \xi)+\sum_{k=1}^{j} w_{k}^{j-k}(x, x, \xi, \xi) .
$$

D'après $(6.10)_{k}$, pour $1 \leq k \leq j$ on a

$$
\begin{aligned}
w_{k}^{j-k}(x, y, \xi, \eta) & =\frac{1}{k}\left[\left(\partial_{\xi} \cdot \partial_{y}\right) w_{k-1}^{j-k}(x, y, \xi, \eta)\right. \\
+ & \left.\sum_{\nu=0}^{k-1} \sum_{l=\nu}^{j-k+\nu} \partial_{\xi} w_{\nu}^{l-\nu}(x, y, \xi, \eta) \cdot \partial_{y} w_{k-1-\nu}^{j-l-k+\nu}(x, y, \xi, \eta)\right] .
\end{aligned}
$$

Si l'on pose $y=x, \eta=\xi$, on a par récurrence, pour $j \geq k \geq 1$,

$$
w_{k}^{j-k}(x, x, \xi, \xi)=\Phi_{k}\left(p_{0}, p_{1}, \ldots, p_{j-1}, q_{0}, q_{1}, \ldots, q_{j-1}\right)
$$

avec un polynôme différentiel $\Phi_{k}$ de $p_{0}, p_{1}, \ldots, p_{j-1}, q_{0}, q_{1}, \ldots, q_{j-1}$ et donc

$$
\begin{aligned}
\sum_{k=1}^{j} w_{k}^{j-k}(x, x, \xi, \xi) & =\sum_{k=1}^{j} \Phi_{k}\left(p_{0}, p_{1}, \ldots, p_{j-1}, q_{0}, q_{1}, \ldots, q_{j-1}\right) \\
& =: \Phi\left(p_{0}, p_{1}, \ldots, p_{j-1}, q_{0}, q_{1}, \ldots, q_{j-1}\right) .
\end{aligned}
$$

(6.13) se récrit comme

$$
r_{j}(x, \xi)=q_{j}(x, \xi)+\Phi\left(p_{0}, p_{1}, \ldots, p_{j-1}, q_{0}, q_{1}, \ldots, q_{j-1}\right)
$$


pour tout $j \geq 1$. Pour $j=0$, on a par (6.12),

$$
r_{0}(x, \xi)=w_{0}^{0}(x, x ; \xi, \xi)=p(x, \xi)+q_{0}(x, \xi) .
$$

D'après (6.15) et (6.16), à partir de $p$ et $\left(r_{j}\right)$, on peut déterminer $\left(q_{j}\right)$.

On peut supposer que $p(x, \xi)$ et $r_{j}(x, \xi)$ satisfont à $(6.2)-(6.6)$ et par (6.7), ils satisfont à la condition suivante pour $\mathfrak{M}$ et 0 respectivement : pour tout ensemble conique $\Omega^{\prime} \subset \Omega$ et $\delta>0$, il existe $d>r$ tel que pour tout $\varepsilon>0$ on ait, avec $C_{\varepsilon}>0$,

$$
\begin{aligned}
& \left|p(x, \xi)-H_{M(x)}(\xi)\right| \leq \varepsilon|\xi|+C_{\varepsilon} \\
& \left|r_{0}(x, \xi)\right| \leq \varepsilon|\xi|+C_{\varepsilon} \quad \text { sur } \Omega^{\prime}[d] \\
& \left|r_{j}(x, \xi)\right| \leq \delta^{j}\left(\varepsilon|\xi|+C_{\varepsilon}\right) \quad \operatorname{sur} \Omega^{\prime}[(j+1) d](j=1,2, \ldots) .
\end{aligned}
$$

Maintenant nous prouvons que pour tous $\varepsilon>0, j, k$ avec $j \geq k$ et tous $(x, \xi),(y, \eta) \in \Omega^{\prime}[(j+1) d]$ avec $|\xi| \geq|\eta|$ et $|x|=|y|$, on a, avec $C_{\varepsilon}>0$,

$$
\left|w_{k}^{j-k}(x, y, \xi, \eta)\right| \leq 2 \delta^{j}\left[H_{x} \cdot|\xi|+\varepsilon|\xi|+C_{\varepsilon}\right] .
$$

Dans le cas de $j=k=0$, on a, d'après (6.12) et (6.16)-(6.18),

$$
\begin{aligned}
\left|w_{0}^{0}(x, y, \xi, \eta)\right| & =\left|p(x, \xi)+q_{0}(y, \eta)\right|=\left|p(x, \xi)+\left(r_{0}(y, \eta)-p(y, \eta)\right)\right| \\
& \leq 2\left(H_{x} \cdot|\xi|+\varepsilon|\xi|+C_{\varepsilon}\right) .
\end{aligned}
$$

Ensuite, on suppose que $j=k \geq 1$; alors d'après (6.14) et l'hypothèse de récurrence, en utilisant la formule de Cauchy, pour tout $\varrho>0$ et petit $\lambda>0$ on a

$$
\begin{aligned}
\left|w_{k}^{0}(x, y, \xi, \eta)\right| & \\
= & \frac{1}{k}\left|\left(\partial_{\xi} \cdot \partial_{y}\right) w_{k-1}^{0}(x, y, \xi, \eta)+\sum_{\nu=0}^{k-1} \partial_{\xi} w_{\nu}^{0}(x, y, \xi, \eta) \cdot \partial_{y} w_{k-1-\nu}^{0}(x, y, \xi, \eta)\right| \\
\leq & \frac{\delta^{k-1}}{k}\left[\frac{1}{\lambda|x| \varrho|\xi|} 2\left[H_{(1+\lambda) x} \cdot((1+\varrho)|\xi|)+\varepsilon(1+\varrho)|\xi|+C_{\varepsilon}\right]\right. \\
& \quad+\sum_{\nu=0}^{k-1} \frac{4}{\lambda|x| \varrho|\xi|}\left[H_{x} \cdot((1+\varrho)|\xi|)+\varepsilon(1+\varrho)|\xi|+C_{\varepsilon}\right] \\
\quad & \left.\times\left[H_{(1+\lambda) x} \cdot|\xi|+\varepsilon|\xi|+C_{\varepsilon}\right]\right] \\
\leq & 2 \delta^{k-1}\left[H_{x} \cdot|\xi|+\varepsilon|\xi|+C_{\varepsilon}\right] \cdot \frac{(1+\lambda)(1+\varrho)}{k \lambda|x| \varrho|\xi|}\left(1+2 k\left[H_{x} \cdot|\xi|+\varepsilon|\xi|+C_{\varepsilon}\right]\right) .
\end{aligned}
$$


On a

$$
\begin{aligned}
\frac{(1+\lambda)(1+\varrho)}{k \lambda|x| \varrho|\xi|}( & \left.1+2 k\left[H_{x} \cdot|\xi|+\varepsilon|\xi|+C_{\varepsilon}\right]\right) \\
\leq & \frac{(1+\lambda)(1+\varrho)}{\lambda \varrho}\left(\frac{1}{k|x||\xi|}+2\left[\frac{H_{x}}{|x|}+\frac{\varepsilon}{|x|}+\frac{C_{\varepsilon}}{|x||\xi|}\right]\right)
\end{aligned}
$$

et prenant $d \gg 1$ et $|\xi|>d$ et prenant $\varepsilon>0$ assez petit, si $|x|$ est assez petit, grâce à (6.1), le membre droit ci-dessus est plus petit que $\delta$, d'où (6.20) pour $j=k$. Pour $j, k$ général avec $j \geq k$, compte tenu de (6.14), par le même raisonnement que dans le cas de $j=k$, on a (6.20).

D'après $(6.13),(6.18)$ et $(6.20)$, on a

$$
\left|q_{0}(x, \xi)-I_{-M(x)}(\xi)\right|=\left|r_{0}(x, \xi)-\left(p(x, \xi)-H_{M(x)}(\xi)\right)\right| \leq 2\left(\varepsilon|\xi|+C_{\varepsilon}\right),
$$

et pour $j \geq 1$, d'après $(6.19)$ on a

$$
\begin{aligned}
\left|q_{j}(x, \xi)\right| & =\left|r_{j}(x, \xi)-\sum_{k=1}^{j} w_{k}^{j-k}(x, x, \xi, \xi)\right| \\
& \leq \delta^{j}\left(\varepsilon|\xi|+C_{\varepsilon}\right)+2(j-1) \delta^{j}\left[H_{x} \cdot|\xi|+\varepsilon|\xi|+C_{\varepsilon}\right]
\end{aligned}
$$

pour $\delta>0$ assez petit, cela est majoré par $3 \delta^{j}\left(\varepsilon|\xi|+C_{\varepsilon}\right)$.

DÉfinition 6.4. Un opérateur pseudo-différentiel $P$ défini en un point $p \in T^{*} X$ est dit non-caractéristique en $p$ s'il existe une famille de supports $\mathfrak{M}=(M(x))$ satisfaisant à $(6.1)$ et $H_{c x} \leq c H_{x}$ pour tout $c>0$, un symbole $P(x, \xi) \in S^{\mathfrak{M}}(\Omega)$, un voisinage conique $\Omega^{\prime} \subset \Omega$ de $p$ et $r^{\prime}>r$ tels que

(6.21) pour tout $\varepsilon>0$, il existe $C_{\varepsilon}>0$ tel que $|P(x, \xi)| \geq C_{\varepsilon} e^{H_{M(x)}(\xi)-\varepsilon|\xi|}$ pour tout $(x, \xi) \in \Omega^{\prime}\left(r^{\prime}\right)$.

ThÉORÈme 6.5. Soit $P \in \mathcal{E}_{[p]}^{\mathbb{R}}$ un opérateur pseudo-différentiel défini en un point $p$. On suppose que $P$ soit non-caractéristique en $p$. Alors dans l'anneau $\mathcal{E}_{[p]}^{\mathbb{R}}$, il y a l'inverse $P^{-1}$ de $P$.

Démonstration. D'abord on suppose qu'il existe un symbole $p(x, \xi) \in$ $S^{0}(\Omega)$ du type $\mathfrak{M}^{+}$tel que $P(x, \xi)=e^{p(x, \xi)}$ dans $\Omega(r)$. D'après la proposition précédente, il existe un symbole formel $q(t ; x, \xi) \in \widehat{S}^{0}(\Omega)$ tel que $Q(t ; x, \xi):=e^{q(t ; x, \xi)}$. Si on désigne par $Q$ l'opérateur correspondant, alors formellement $\sigma(P \circ Q)=1$, c'est-à-dire, dans la notation de la démonstration de la proposition 6.3 ,

$$
\begin{aligned}
& q_{0}(x, \xi)=-p(x, \xi) \\
& q_{j}(x, \xi)=-\sum_{k=1}^{j} w_{k}^{j-k}(x, x, \xi, \xi) \quad(j \geq 1) .
\end{aligned}
$$


Puisque le symbole formel $q(t ; x, \xi)=\sum_{j=0}^{\infty} t^{j} q_{j}(x, \xi)$ ainsi obtenu est du type $(-\mathfrak{M})^{-}, Q(t ; x, \xi)$ est bien un opérateur pseudo-différentiel défini en $p$.

Dans le cas général, en utilisant la proposition 5.5, on peut déterminer formellement $Q(t ; x, \xi)=\sum_{j=0}^{\infty} t^{j} Q_{j}(x, \xi)$ par la formule

$$
\begin{aligned}
Q_{0}(x, \xi) & :=\frac{1}{P(x, \xi)}, \\
Q_{k}(x, \xi) & :=-\frac{1}{P(x, \xi)} \sum_{|\alpha|=0}^{k} \frac{1}{\alpha !} \partial_{\xi}^{\alpha} P(x, \xi) \partial_{x}^{\alpha} Q_{k-|\alpha|}(x, \xi) \quad(k \geq 1),
\end{aligned}
$$

et d'après le raisonnement ci-dessus pour $Q(t ; x, \xi)$ du type spécial $e^{q(t ; x, \xi)}$, on doit avoir

$$
Q(t ; x, \xi)=\frac{1}{P(x, \xi)} e^{\sum_{j=1}^{\infty} t^{j} q_{j}(x, \xi)},
$$

où d'après la démonstration de la proposition 6.3 , chaque $q_{j}(x, \xi)(j \geq 1)$ est un polynôme différentiel de $\partial_{x} p(x, \xi)=\partial_{x} P(x, \xi) / P(x, \xi)$ et $\partial_{\xi} p(x, \xi)=$ $\partial_{\xi} P(x, \xi) / P(x, \xi)$. Par conséquent, compte tenu du lemme 6.12 (et de la preuve de (6.12)) de [7], le raisonnement ci-dessus marche aussi pour le cas général. D'où l'on a un opérateur $Q$ tel que $\sigma(P \circ Q)=1$.

Par le même raisonnement, on peut trouver un opérateur $Q^{\prime}$ tel que $\sigma\left(Q^{\prime} \circ P\right)=1$ et enfin $Q^{\prime}=Q$ est bien l'inverse de $P$.

\section{Exemples : opérateurs micro-différentiels en un point et opé-} rateurs différentiels en un point. Dans les conditions de la définition 3.4, soit $Z \subset X \times X$ un $\mathfrak{M}$-ordinal. L'espace $X \times X$ est muni d'une action de $\mathbb{C}_{\times}$définie par $c \cdot(x, y):=(c \cdot x, c \cdot y)$ pour $c \in \mathbb{C}_{\times}$et $(x, y) \in X \times X$. L'action de $c$ est une rotation si $|c|=1$. Un ensemble dans $X$ ou bien $X \times X$ est dit de Reinhardt s'il est invariant par toute rotation. De même, on définit la $\mathbb{C}$-action sur $X \times X$.

DÉfinition 7.1. Soit $Z$ de Reinhardt. Pour tous voisinages ouverts $D_{1}$ et $D_{2}$, on appelle $P \in \mathcal{E}\left(Z ; D_{1}, D_{2}\right)$ un opérateur micro-différentiel défini en $p$. On pose

$$
\mathcal{E}_{[p]}^{\infty}:=\underline{\lim } \mathcal{E}\left(Z ; D_{1}, D_{2}\right),
$$

où la limite inductive est prise pour des familles de supports $\mathfrak{M}=(M(x))$ avec $M(x)$ contenant $0, \mathfrak{M}$-ordinaux $Z$ qui sont de Reinhardt et des voisinages ouverts $D_{1}, D_{2}$ de $x_{0}$.

Dans cette section, pour tout $P \in \mathcal{E}\left(\mathcal{G} ; D_{1}, D_{2}\right)$, où $\mathcal{G}=(G(x)), G(x)=$ $\bigcap\left(\Gamma_{k}+M(x)\right), \Gamma_{k}$ étant de la forme

$$
\begin{aligned}
\Gamma_{1} & :=\left\{z \in X \mid z_{1} \neq 0\right\}, \\
\Gamma_{j} & =\Gamma_{j, \delta}:=\left\{z \in X|\delta| z_{j}|\leq| z_{1} \mid\right\} \quad(2 \leq j \leq n)
\end{aligned}
$$


avec $\delta>0$, on calcule explicitement le noyau (4.10). Soit $K(x, y) d y$ un des noyaux de $P$. Par le même raisonnement que dans [16], remarquons que dans ce cas-là, $K$ peut se développer en série :

$$
K(x, y)=\sum_{\alpha=\left(\alpha_{1}, \alpha_{2}, \ldots, \alpha_{n}\right) \in \mathbb{Z}^{n}, \alpha_{2}, \ldots, \alpha_{n} \geq 0} a_{\alpha}(x) \Phi_{\alpha}(x-y)
$$

où $\Phi_{\alpha}(z)$ est défini dans [16, p. 337], comme suit : pour $\nu \in \mathbb{Z}$,

$$
\Phi_{\nu}(\tau):= \begin{cases}\frac{1}{2 \pi \sqrt{-1}} \frac{\nu !}{(-\tau)^{\nu+1}} & (\nu \geq 0), \\ -\frac{1}{2 \pi \sqrt{-1}} \frac{\tau^{-\nu-1}}{(-\nu-1) !}\left(\ln \tau-\left(\sum_{q=1}^{-\nu-1} \frac{1}{q}-\gamma\right)\right) & (\nu<0)\end{cases}
$$

et $\Phi_{\alpha}(z):=\Phi_{\alpha_{1}}\left(z_{1}\right) \cdots \Phi_{\alpha_{n}}\left(z_{n}\right)$ (où $\gamma$ est la constante d'Euler). Dans ce cas-là, considérons la série formelle

$$
\widehat{P}(x, \xi)=\sum_{\alpha=\left(\alpha_{1}, \alpha_{2}, \ldots, \alpha_{n}\right) \in \mathbb{Z}^{n}, \alpha_{2}, \ldots, \alpha_{n} \geq 0} a_{\alpha}(x) \xi^{\alpha}=\sum_{i=-\infty}^{\infty} \widehat{P}_{i}(x, \xi)
$$

et appelons-la le symbole formel de $P \in \mathcal{E}\left(\mathcal{G} ; D_{1}, D_{2}\right)$, où l'on a posé $\widehat{P}_{i}(x, \xi)$ $:=\sum_{|\alpha|=i} a_{\alpha}(x) \xi^{\alpha}$, la "partie homogène" d'ordre $i$ par rapport à $\xi$. Nous remarquons que la partie negative (i.e. $i<0$ ) du symbole formel n'est pas le vrai symbole au sens de la proposition 4.2. En effet, le symbole formel ne converge pas pour $i<0$, en général. Comme un type spécial d'un opérateur micro-differéntiel en un point, on définit un opérateur différentiel :

DÉFINITION 7.2. On définit la classe des opérateurs différentiels définis en $x_{0}$ par

$$
\mathcal{D}_{\left[x_{0}\right]}^{\infty}:=\lim _{\longrightarrow} H_{\Delta_{\mathfrak{M}}}^{n}\left(D_{1} \times D_{2}, \mathcal{O}_{X \times X}^{(0, n)}\right)
$$

où la limite inductive est prise pour des voisinages ouverts $D_{1}, D_{2}$ de $x_{0}$.

$\mathcal{D}_{[p]}^{\infty}$ est juste la classe des opérateurs micro-différentiels ayant la partie positive d'indexe dans (7.1), qui coïncide à la classe définie dans [7]:

Proposition 7.3. On a l'identification canonique suivante :

$$
\mathcal{D}_{\left[x_{0}\right]}^{\infty} \simeq\left\{P \in \mathcal{E}_{[p]}^{\infty} \mid P \text { a le symbole de la forme } P(x, \xi)=\sum_{\alpha \geq 0} a_{\alpha}(x) \xi^{\alpha}\right\} .
$$

EXemple. Supposons $n=1$; alors l'indicatrice (4.4) coïncide à $h_{P, x}(\xi)$. Considérons des opérateurs $P(x, D)=\exp (x D)$ et $Q(x, D)=\exp (-x D)$ ayant leurs symboles $P(x, \xi)=\exp (x \xi)$ et $Q(x, \xi)=\exp (-x \xi)$. Ces opérateurs ne sont ni des opérateurs différentiels locaux au sens de [16], ni des opérateurs différentiels non-locaux au sens de [9], mais ils sont des opérateurs 
différentiels en le point $x_{0}=0$. En effet, pour tous $x$ et $\xi$ on a

$$
|P(x, \xi)|=e^{\operatorname{Re} x \xi} \leq e^{|x||\xi|}, \quad|Q(x, \xi)|=e^{-\operatorname{Re} x \xi} \leq e^{|x||\xi|}
$$

et leurs indicatrices de croissance sont, respectivement, $h_{P, x}(\xi)=\operatorname{Re} x \xi$, $h_{Q, x}(\xi)=-\operatorname{Re} x \xi$. Pour toute fonction holomorphe $f(x)$ définie en 0 , on a

$$
P(x, D) f(x)=f(2 x), \quad Q(x, D) f(x)=f(0),
$$

donc $P$ a l'inverse $P^{-1}=\exp (-(x \xi) / 2)$ dans $\mathcal{D}_{[0]}^{\infty}$, tandis que $Q$ n'a pas d'inverse dans la même classe.

Malheureusement, ces exemples ne satisfont pas à la condition (6.1) du théorème 6.5 et donc on ne peut pas construire leurs inverses à la manière de la théorie développée dans le présent article.

Exemple. Supposons $n=1$ et $m>1$. Considérons l'opérateur $P(x, D)$ $=\exp \left(x^{m} D\right)$ à symbole $P(x, \xi)=\exp \left(x^{m} \xi\right)$. Dans ce cas-là, en prenant $M(x):=\left\{x^{m}\right\}$, la fonction d'appui $H_{M(x)}(\xi)$ satisfait bien à (6.1). En effet, on a $H_{M(x)} \leq|x|^{m}|\xi|$. Pour $p=\left(x_{0}, \xi_{0}\right)$ quelconque, $P$ est bien-entendu noncaractéristique en $p$, et donc $P$ admet l'inverse dans l'anneau des opérateurs pseudo-différentiels $\mathcal{E}_{[p]}^{\mathbb{R}}$ en $p$.

\section{Références}

[1] T. Aoki, Invertibility for microdifferential operators of infinite order, Publ. RIMS Kyoto Univ. 18 (1982), no. 2, 1-29.

[2] -, Calcul exponentiel des opérateurs microdifférentiels d'ordre infini I, Ann. Inst. Fourier (Grenoble) 33 (1983), no. 4, 227-250.

[3] —, The Symbolical Theory of Pseudo-Differential Operators of Infinite Order, Lecture Notes in Math. Sci. 14, Tokyo Univ., 1997 (noté par S. Yamazaki) (en Japonais).

[4] T. Aoki, K. Kataoka et S. Yamazaki, Hyperfonctions, FBI Transformation, Opérateurs Pseudo-Différentiels d'Ordre Infini, Kyouritu Shuppan, 2004 (en Japonais).

[5] L. Hörmander, An Introduction to Complex Analysis in Several Variables, Van Nostrand Reinhold, 1966.

[6] - The Analysis of Linear Partial Differential Operators III, Grundlehren Math. Wiss. 274, Springer, Berlin, 1985.

[7] R. Ishimura, Opérateurs différentiels d'ordre infini définis en un point, Mem. Fac. Sci. Kyushu Univ. 40 (1986), 31-39.

[8] —, Endomorphismes de l'espace des germes de fonctions holomorphes en un point et opérateurs différentiels d'ordre infini, Ann. Polon. Math. 49 (1988), 129-133.

[9] —, Non-local pseudo-differential operators, J. Math. Pures Appl. 81 (2002), 12411276 .

[10] - Existence and continuation of analytic solutions of the non-local differential equations, preprint.

[11] R. Ishimura, J. Okada and Y. Okada, Continuation of holomorphic solutions to convolution equations in complex domains, Ann. Polon. Math. 74 (2000), 105-115.

[12] M. Kashiwara and P. Schapira, Micro-hyperbolic systems, Acta Math. 142 (1979), $1-55$. 
[13] M. Kashiwara and P. Schapira, Sheaves on Manifolds, Grundlehren Math. Wiss. 292, Springer, 1990.

[14] P. Lelong and L. Gruman, Entire Functions of Several Complex Variables, Grundlehren Math. Wiss. 282, Springer, Berlin, 1986.

[15] L. I. Ronkin, Functions of Completely Regular Growth, Math. Appl. (Soviet Ser.) 81, Kluwer, 1992.

[16] M. Sato, T. Kawai and M. Kashiwara, Microfunctions and pseudo-differential equations, in: Lecture Notes Math. 287, Springer, 1973, 265-529.

[17] B. Sternin and V. Shatalov, Differential Equations on Complex Manifolds, Math. Appl. 276, Kluwer, 1994.

Département de Mathématiques et Informatique

Faculté des Sciences

Université de Chiba

Yayoicho, Chiba, 263-8522 Japon

E-mail: ishimura@math.s.chiba-u.ac.jp

Received 16.11.2005

and in final form 18.3.2006 